\title{
DEVELOPMENT OF A STATIC IN VITRO DIGESTION MODEL FOR DETERMINATION OF GLYCEMIC INDEX
}

\author{
by \\ Yesudas Gudivada \\ B.Sc., Ryerson University, Toronto, Canada, 2014
}

\author{
A thesis presented to Ryerson University in \\ partial fulfillment of the requirements for the degree of \\ Master of Science \\ in the program of \\ Molecular Science
}

Toronto, Ontario, Canada, 2017

CCopyright by Yesudas Gudivada 2017 


\section{AUTHOR'S DECLARATION FOR ELECTRONIC SUBMISSION OF A DISSERTATION}

I hereby declare that I am the sole author of this dissertation. This is a true copy of the dissertation, including any required final revisions, as accepted by my examiners.

I authorize Ryerson University to lend this dissertation to other institutions or individuals for the purpose of scholarly research.

I further authorize Ryerson University to reproduce this dissertation by photocopying or by other means, in total or in part, at the request of other institutions or individuals for the purpose of scholarly research.

I understand that my dissertation may be made electronically available to the public. 
DEVELOPMENT OF A STATIC IN VITRO DIGESTION MODEL FOR DETERMINATION OF GLYCEMIC

\author{
Yesudas Gudivada \\ Master of Science, 2017 \\ Molecular Science, Ryerson University
}

\title{
Abstract
}

While in vivo methods have been used to determine the glycemic response of food, they are time consuming, costly, and not suitable for large-scale applications. As an alternative, in vitro digestion models offer fast, reproducible results to study food digestion kinetics that are less expensive than conducting human trials. While there are several in vitro glycemic index (GI) methods used to determine the Gl of food, most do not employ methods of in vivo testing. Therefore, we used a static in vitro digestive system, the Dedicated Ryerson University In-vitro Digester (DRUID), that simulates both gastric and intestinal conditions to determine the glycemic response of commonly consumed carbohydrate-containing foods. Samples were collected at regular intervals over a $2 \mathrm{~h}$ residence time after digestion in the intestinal phase of the DRUID. The DRUID-determined GI values were compared to published in vivo GI values. A Bland-Altman plot showed that there was agreement between the GI values determined from the DRUID compared with published in vivo GI values. In conclusion, the in vitro DRUID can reliably and reproducibly determine the GI across a spectrum of carbohydrate-containing foods, and has the potential to predict the digestion kinetics of novel food products in vivo that may promote human health. 


\section{Acknowledgements}

I would like to express my sincere gratitude to my co-supervisors, Dr. Rousseau and Dr. Bellissimo for their continuous support, knowledge, understanding, enthusiasm and humour. This was an amazing experience and I am very thankful and grateful to Dr. Rousseau and Dr. Bellissimo for giving me this wonderful opportunity. Thank you for your patience and guidance that you both have given me. Thank you.

My sincerest gratitude also goes to all of my lab-mates for making my experience in the Food and SMaRT lab a very memorable one. I enjoyed everyone's company and I am so thankful that everyone was so friendly and nice. I am especially grateful for the help I received from Dr. Nicole Green for my study with the confocal microscope. I am also grateful for the friendship, help and kindness shown by Ryan West. Thank you. I would also like to convey my thanks to Ruby Rafanan for her guidance and amazing advice. Thank you.

Having a co-supervisor meant that I was fortunate enough to meet another group of great people. This is of course the students in Dr. Bellissimo's First and NeXT Labs. Thank you all for accepting me and sharing your friendship. I would especially like to thank Stella Paterakis for being a great friend and co-worker. Thank you.

I would also like to thank the managers of the Architectural department at Ryerson University, Zane and Frank for letting me use their shop and tools and for all their help and assistance in designing the in vitro digestion model, the "DRUID". My sincere gratitude goes to Graham Pearson from the Physics Department at Ryerson University; thank you, Graham for all your time and help and thank you for letting me use the tools in the workshop and for assisting me with designing the DRUID.

I would also like to thank Dr. Antonescu for being an amazing professor, my committee member and for allowing me to use his lab for performing SDS-PAGE and Western Blotting. I am also thankful for his guidance in my project. Thank you, sir.

Finally, I would like to express my very profound gratitude to my family for providing me with unfailing support and continuous encouragement throughout my years of study. This accomplishment would not have been possible without them. Thank you. 


\section{Table of Contents}

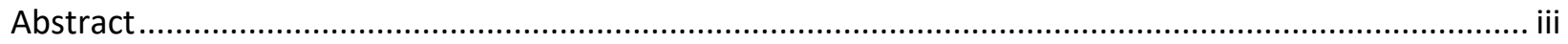

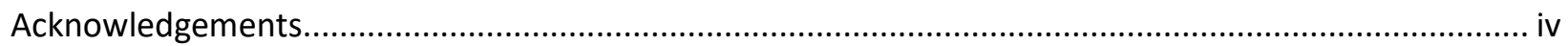

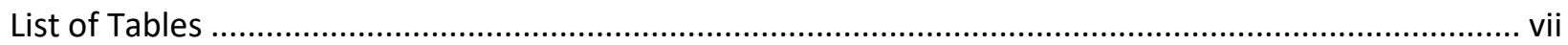

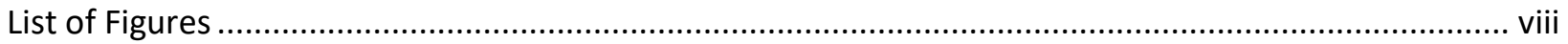

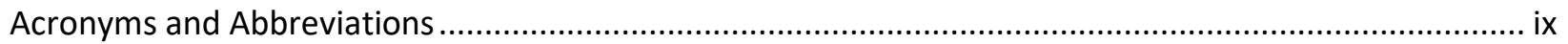

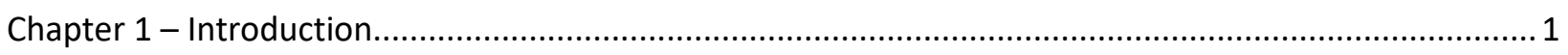

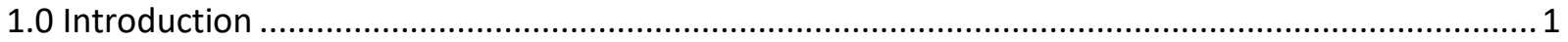

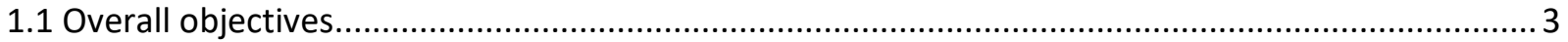

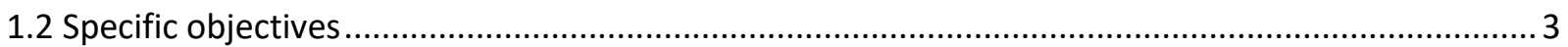

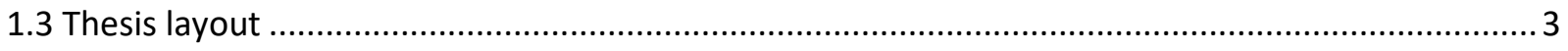

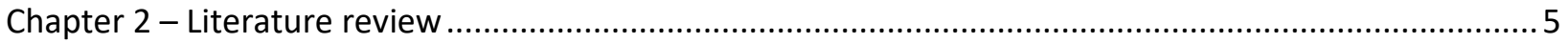

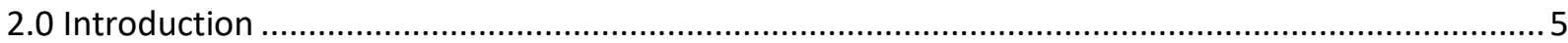

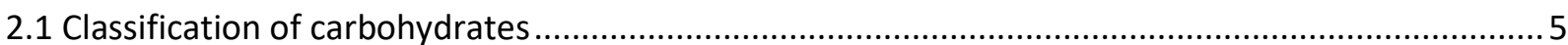

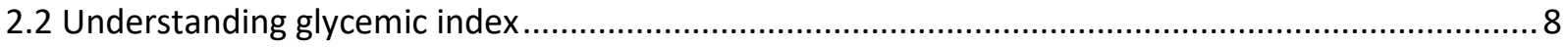

2.2.1 In vivo measurement of the glycemic index ..................................................................... 11

2.3 Anatomy and physiology of the gastrointestinal tract ...............................................................11

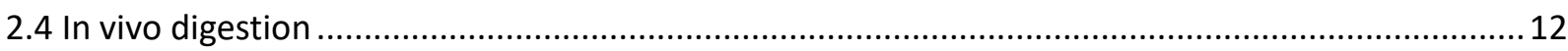

2.4.1 Digestion and absorption of carbohydrates ..................................................................... 17

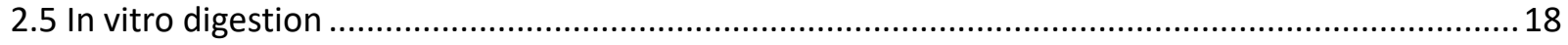

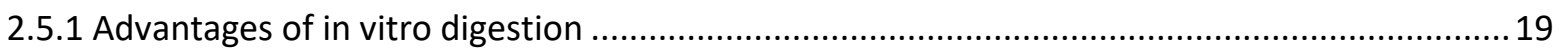

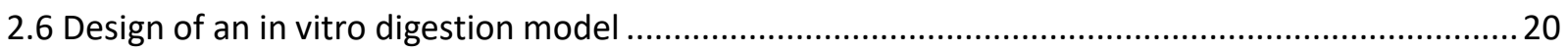

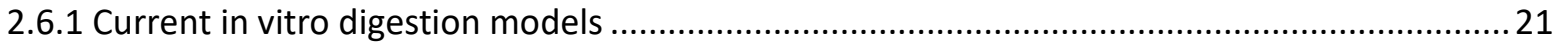

2.7 Considerations for designing an in vitro digestive tract: Stage by stage ......................................23

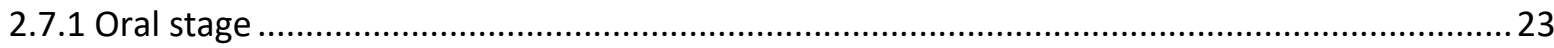

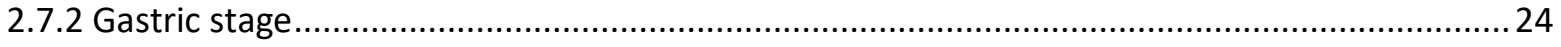

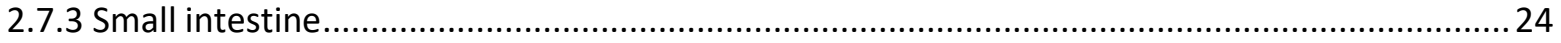

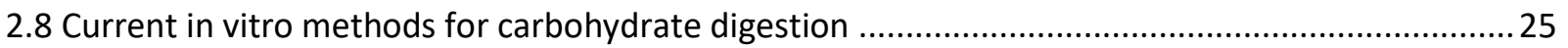

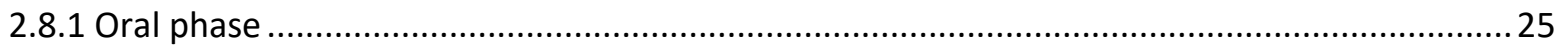

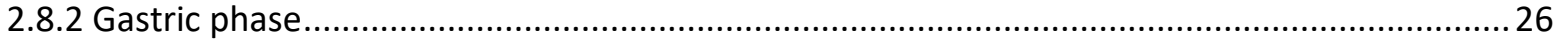

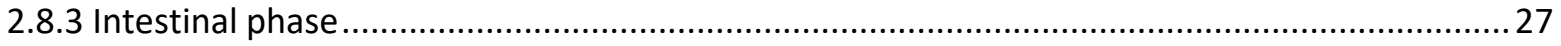

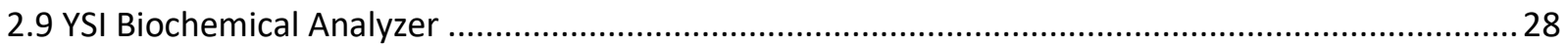




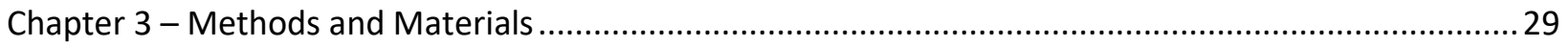

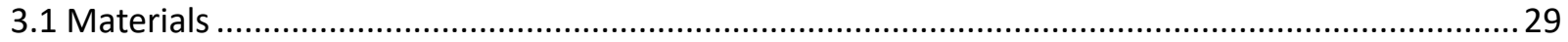

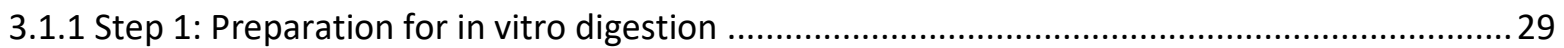

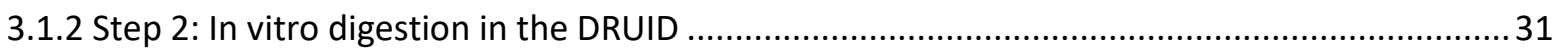

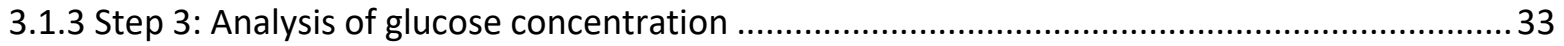

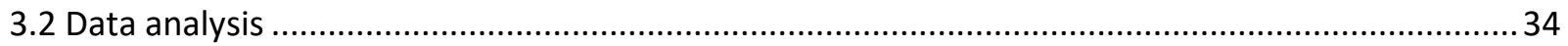

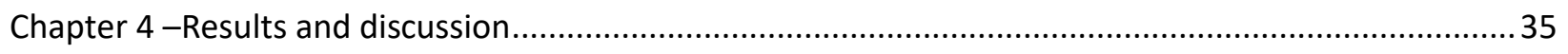

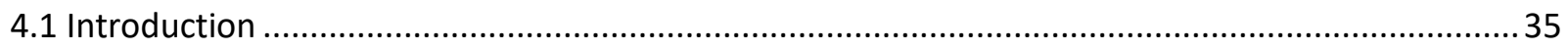

4.2 The DRUID against other in vitro GI determining methods ......................................................45

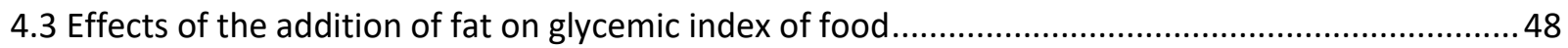

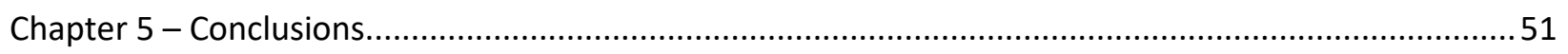

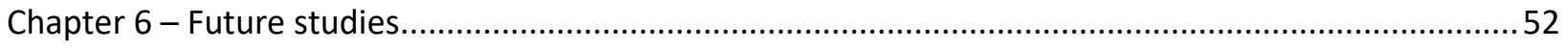

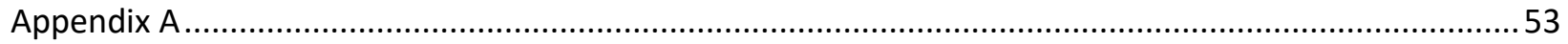

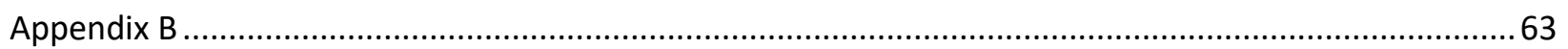

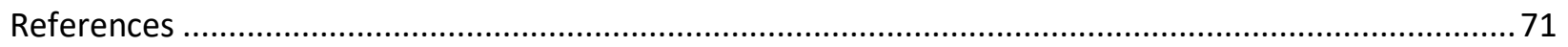




\section{List of Tables}

Table 2.1. Carbohydrate classification based on chemical properties.

Table 2.2. Enzymes of the gastrointestinal tract. Modified from Verkerstaff Jonegan, 2004.

Table 4.1. Comparison of the in vivo and in vitro GI values of a spectrum of foods 42 against white bread (50 g of available carbohydrates). 


\section{List of Figures}

Figure 2.1. Structure of amylose and amylopectin.

Figure 2.2. Mechanical action from the peristaltic contractions of the stomach. Modified from Kong and Singh, 2008.

Figure 4.1. Breakdown of food against white bread (50 g of available carbohydrates) in the small intestinal compartment of the DRUID.

Figure 4.2. Change in glucose concentration of the test and control foods over $2 \mathrm{~h}$ in the small intestine compartment of the DRUID.

Figure 4.3. Use of a Bland-Altman plot to analyze the agreement between the in vivo and in vitro GI determined values.

Figure 4.4. Correlation between the in vitro GI values determined by the DRUID and the published in vivo $\mathrm{Gl}$ values for the tested foods.

Figure 4.5. The effect of fat on the glycemic index of white rice against white bread. 49 Means with the same letter are not significantly different $(p>0.05)$.

Figure 4.6. The effect of fat on the breakdown of white rice against white bread (50 g of 50 available carbohydrates) in the small intestinal compartment of the DRUID. 


\section{Acronyms and Abbreviations}

(In alphabetical order)

\begin{tabular}{|c|c|}
\hline abs & Absorbance \\
\hline $\mathrm{CHO}$ & Carbohydrates \\
\hline Eqn & Equation \\
\hline DGM & Dynamic gastric model \\
\hline g & Gram(s) \\
\hline Gl & Glycemic index \\
\hline GIT & Gastrointestinal tract \\
\hline GOPOD & Glucose oxidase/peroxidase \\
\hline $\mathrm{h}$ & Hour(s) \\
\hline HGS & Human gastric simulator \\
\hline ¡AUC & Incremental area under the curve \\
\hline $\min$ & Minute(s) \\
\hline $\mathrm{mmol} / \mathrm{L}$ & Millimoles per litre \\
\hline PVP & Peristaltic valve pumps \\
\hline RDS & Rapidly digestible starch \\
\hline $\mathrm{rpm}$ & Rotations per minute \\
\hline SD & Standard deviation \\
\hline SDS & Slowly digestible starch \\
\hline TAG & Triacylglycerol \\
\hline$U$ & Units \\
\hline
\end{tabular}




\section{Chapter 1 - Introduction}

\subsection{Introduction}

Carbohydrates $(\mathrm{CHO})$ are the main source of energy in the diets of humans, making up about $40-80 \%$ of caloric intake ${ }^{1}$. The brain, kidneys, and muscles require carbohydrates to function optimally, and to also aid in the synthesis of certain amino acids ${ }^{2}$. Carbohydrates are a family of compounds that are all composed of the same monosaccharide building units. They range from simple sugars, monosaccharide and disaccharides, through oligosaccharides and dextrin, to complex starch and non-starch polysaccharides.

Traditionally, carbohydrate-containing foods have been categorized according to their structural classification ${ }^{3}$. As a result, it was assumed that when "simple" carbohydrates were consumed, it would cause a rapid glucose response in the body thus being unsuitable for diabetics while "complex" carbohydrates were thought to have caused slower and smaller responses to blood glucose thus were believed to be a healthier option for patients with glucose intolerance ${ }^{3}$.

However, a study by Conn and Newburgh (1939) showed that different carbohydrate foods with a similar macronutrient content elicited different glycemic responses ${ }^{4}$. They found that, even though the foods had similar macronutrient composition, there were differences in the rate at which they were metabolized into glucose. Jenkins et al. (1981) later concluded that the glucose response of carbohydrate-containing foods was not only a result of the primary structure of the carbohydrate but also is influenced by the physical form of the carbohydrate (like particle size and degree of hydration) and dietary fibre ${ }^{5}$. All of these factors helped to influence 
the physiological properties (ability to raise blood glucose levels) of the foods consumed and thus were important for understanding the nutritional and health effects of carbohydrates ${ }^{6}$.

In order to incorporate all the factors influencing the physiological properties of carbohydrates, a system was needed to classify the carbohydrate containing food based on its glycemic response ${ }^{7}$. This led to the development of the glycemic index (GI), which is a quantitative measure of how much a carbohydrate-containing food raises blood glucose levels relative to a standard food ${ }^{8}$. It is based on the degree of glucose release (physiological properties) into the bloodstream after consumption of a carbohydrate-containing food ${ }^{9}$.

The Gl of many foods has been determined through traditional in vivo methods. However, in vivo $\mathrm{Gl}$ methods are time consuming, costly, and not suitable for large-scale applications ${ }^{10}$. As an alternative, in vitro digestion models offer fast, reproducible results to study food digestion kinetics that are less expensive than conducting human trials.

While there are several in vitro GI methods that are used to determine the Gl of food, most do not employ methods of in vivo testing and do not simulate the physiological conditions that occur in vivo. The purpose of this research was to develop a static in vitro digestion model called the "Dedicated Ryerson University In-vitro Digester" (DRUID) that simulates both gastric and intestinal conditions to determine the glycemic index of commonly consumed carbohydratecontaining foods. 


\subsection{Overall objectives}

This study investigated how food is digested in an in vitro digestion model that mimics a mammalian in vivo digestive tract.

The objectives were to:

- Develop a static in vitro gastrointestinal model that simulates an in vivo human digestive system.

- Perform a series of tests to validate the DRUID for GI determination.

\subsection{Specific objectives}

1. Validate the model for determining the in vitro glycemic index of commonly consumed carbohydrate-containing foods (ranging from low to high GI).

2. Examine the effect of different amounts of fat on the $\mathrm{Gl}$ of a carbohydrate containing food.

3. Calculate the available carbohydrates of foods by determining the glucose, fructose, sucrose and starch content of foods without a manufacturer's nutritional label.

\subsection{Thesis layout}

The thesis is presented in 9 chapters as follows:

\section{Chapter 1: Introduction}

Chapter 1 presents an introduction to the study. It includes a summary of carbohydrates, the concept and development of GI and the development of a static in vitro digestion model for determining the $\mathrm{Gl}$ of carbohydrate-containing food. The problem 
statement and motivation behind the development of an in vitro digestion model are also stated along with the objectives of the project.

\section{Chapter 2: Literature review}

In chapter 2, the background and nutritional significance of carbohydrates, $\mathrm{Gl}$ and in vitro and in vivo digestion methods are discussed. Current in vitro digestion models are introduced. This chapter also includes steps to designing an in vitro digestion model.

\section{Chapter 3: Methods and Materials}

Chapter 3 presents the methodology for determining the $\mathrm{Gl}$ of carbohydrate containing food after digestion in the DRUID. This chapter introduces the selected test foods that were used for the determination of GI in vitro. This chapter also introduces the development of the DRUID and the different compartments of the DRUID.

\section{Chapter 4: Results and discussion}

In chapter 4 the assessment of the DRUID as an in vitro Gl determination model is explored. Results from the determination of GI after digestion in the DRUID were compared to in vivo data from previous literature. A Bland-Altman plot was used to show the agreement between the in vitro and in vivo $\mathrm{Gl}$ values of the selected foods. The in vitro determination of $\mathrm{Gl}$ using the DRUID was compared with other GI determining methods. This chapter also presents a study on how fat affects the GI of white rice.

\section{Chapter 5: Conclusion}

In this concluding chapter, major findings of the project were summarized. This chapter also describes potential applications of the DRUID for determining GI. 


\section{Chapter 6: Future studies}

This chapter presents the potential of the DRUID for future research in the in vitro determination of GI.

\section{Chapter 2 - Literature review}

\subsection{Introduction}

The focus of this chapter is to provide a background and to show the significance of carbohydrates, $\mathrm{Gl}$ and in vivo and in vitro digestion. This chapter also introduces the major in vitro digestion models that are currently available.

\subsection{Classification of carbohydrates}

Carbohydrates $(\mathrm{CHO})$ are classified by their monomer composition, molecular size, degree of polymerization and type of linkages ${ }^{11}$. This classification divides carbohydrates into four groups: monosaccharides, disaccharides, oligosaccharides and polysaccharides (Table 2.1) ${ }^{12}$. The most common monosaccharides include glucose, fructose and galactose ${ }^{12}$. Disaccharides consist of two sugar monosaccharides linked via a glycosidic bond and common examples include sucrose (glucose + fructose), lactose (galactose + glucose) and maltose (glucose + glucose $)^{12}$. Oligosaccharides consists of three to nine glycosidic bond-linked residues that are water soluble. They are generally resistant to digestion in the upper digestive tract but are fermented in the large intestine by the microflora ${ }^{12}$. Polysaccharides have a degree of polymerization that is greater than nine ${ }^{12}$. There are two main type of dietary polysaccharides that are important in human nutrition: starch and non-starch polysaccharides (such as dietary fibre). 
Table 2.1. Carbohydrate classification based on chemical properties. Modified from FAO/WHO, 1998.

\begin{tabular}{|l|l|}
\hline \multicolumn{2}{|l|}{ Carbohydrates } \\
\hline $\begin{array}{l}\text { Monosaccharides } \\
\text { (1 monosaccharide) }\end{array}$ & Glucose, Fructose and Galactose \\
\hline $\begin{array}{l}\text { Disaccharides } \\
(2 \text { monosaccharides })\end{array}$ & Sucrose, Lactose, and Maltose \\
\hline $\begin{array}{l}\text { Oligosaccharides } \\
(3-9 \text { monosaccharides) }\end{array}$ & Raffinose, Stachyose \\
\hline $\begin{array}{l}\text { Polysaccharides } \\
\text { (10 or more monosaccharides) }\end{array}$ & Starch, glycogen \\
\cline { 2 - 2 } & Non-starch polysaccharides: Cellulose \\
\hline
\end{tabular}

\subsubsection{Starch}

Starch is the dominant storage carbohydrate in plants such as cereals, seeds and legumes. Starch consists of two main types of macromolecules, amylose and amylopectin ${ }^{13}$. Amylose is a long, unbranched, helical chain containing 500-2,000 glucose residues linked by $\alpha-1,4$-glycosidic bonds that makes up $20-30 \%$ of starch (Figure 2.1). Amylopectin is a highly branched structure containing $10^{4}-10^{5}$ glucose residues linked by both $\alpha-1,4$ as well as $\alpha-1,6$-glycosidic linkages ${ }^{14}$ (Figure 2.1). Amylopectin makes up $70-80 \%$ of total starch and is the major form in most starchbased food 15 .

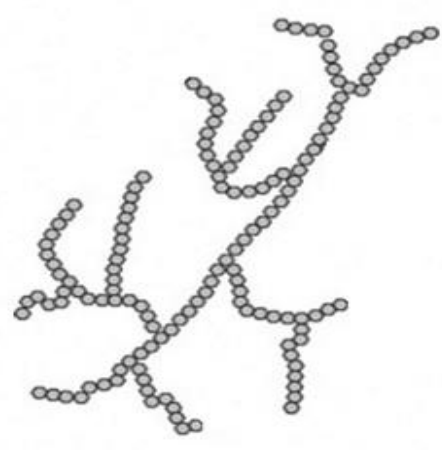

Amylopectin

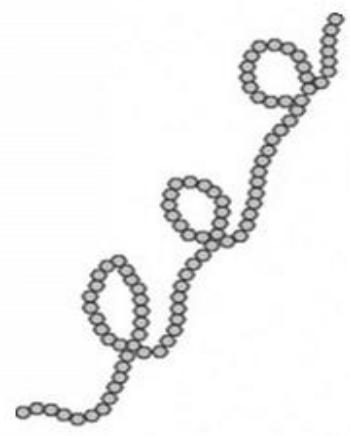

Amylose

Figure 2.1. Structure of amylose and amylopectin. 
The helical structure of amylose arising from the twist after six glucose residues and the hydrogen bonding between the glucose chains confers amylose a compact structure (Figure 2.1). This makes amylose less accessible to enzymatic digestion than the more branched amylopectin ${ }^{14}$. A study by Behall et al. (1988) showed that a meal containing cornstarch with $70 \%$ of amylose resulted in a lower glycemic response than a meal containing cornstarch with $70 \%$ of amylopectin $^{16}$.

Non-starch polysaccharides are the other important type of polysaccharide in the human diet. According to Trowell et al. (1976), non-starch polysaccharides such as dietary fibre are plant cell wall material that do not get digested in the small intestine due to a lack of appropriate digestive enzymes ${ }^{17}$. They are made up of long chains of glycosidic bond-linked monosaccharides that are not always glucose residues ${ }^{17}$. One example of a non-starch polysaccharide is cellulose, which is the structural polysaccharide of the plant cell wall. It is made of $\beta-1,4$-glycosidic bondlinked glucose residues. It is able to form beta sheets via the higher bonds and thus is highly resistant to degradation ${ }^{18}$. Hemicelluloses are branched heteropolymers made up of an array of monomers including glucose, mannose, arabinose and $x y l o s e^{18}$.

\subsubsection{Nutritional classification of carbohydrates}

Available carbohydrates are the portion of dietary carbohydrates that can be metabolized by the body ${ }^{19}$. These include soluble sugars and starch (which are digested and absorbed by the body) but exclude dietary fibre ${ }^{20}$. In contrast, unavailable carbohydrates are the portion of carbohydrates (including dietary fibre like cellulose, hemicellulose and pectin) that are not 
digested by the small intestine but provide the body with energy following fermentation by the microbiota in the large intestine ${ }^{21}$.

Carbohydrates can also be classified by a nutritional factor as proposed by Englyst et al. $(1992)^{22}$. One of these factors is known as resistant starch. Resistant starch (RS) is defined as the fraction of starch and as well as its byproducts that are not absorbed by the small intestine ${ }^{18}$. Englyst defined the term rapidly digestible starch (RDS) and slowly digestible starch (SDS ${ }^{22}$. RDS are found in cooked bread and potatoes and these are rapidly digested in the small intestine within $20 \mathrm{~min}^{22}$. On the other hand, SDS which are found in seeds and grains are slowly digested in the small intestine (between 20 and $120 \mathrm{~min}$ ) since these foods hinder access as a physical barrier for enzymatic digestion 22 .

\subsection{Understanding glycemic index}

The concept of glycemic index (GI) was developed by Jenkins (1981) to classify carbohydrate-containing foods according to their physiological properties (which are not evident just by the structural composition of carbohydrates) $)^{5}$. The $\mathrm{GI}$ is measured by determining the incremental area under the blood glucose response curve after the consumption of a test food containing $50 \mathrm{~g}$ of available carbohydrates and expressed as a percentage of the response to an equivalent carbohydrate portion of a reference food (either glucose or white bread) taken by the same individual ${ }^{11}$. The $\mathrm{Gl}$ is calculated as follows:

$$
G I=\frac{i A U C \text { of test food }}{\text { iAUC of reference food }} \times 100
$$

where iAUC stands for incremental area under the curve. 
This method indexes food on a scale from $0-100$. Low Gl foods such as apples and beans have values below 55 whereas medium GI foods such as whole wheat have values of 56-69. High GI foods such as white bread and processed cereals have $\mathrm{GI}$ values greater than $70^{23}$. Low GI foods are slowly digested and have gradual release of glucose into the bloodstream ${ }^{23}$. While high Gl foods are quickly digested and lead to a high glycemic response with short duration ${ }^{23}$.

The concentration of glucose in the human body is maintained due to homeostatic equilibrium between insulin and glucagon hormones. The glycemic response is a disruption of this equilibrium due to an increase in the blood glucose concentration after consumption, digestion and absorption of a carbohydrate-containing food. Depending on factors like the type of food consumed can affect how rapidly this change in blood glucose concentration can occur. Slow carbohydrate digestion and absorption leads to a low glycemic response, which is gradual and is long lasting. On the other hand, fast carbohydrate digestion and absorption leads to a high glycemic response, which is high in amplitude, fast and is temporary in duration. There are two main hormones that maintain the homeostatic equilibrium of blood sugar, insulin and glucagon. Insulin is secreted by the $\beta$-cells of the pancreatic islets of Langerhans and glucagon is secreted by the $\alpha$-cells of the pancreatic islets of Langerhans. When blood glucose levels are high (hyperglycemia), the secretion of the hormone insulin is triggered and insulin causes the cells of the body (specifically muscle, adipose and liver tissue cells) to take in the glucose from the blood $^{24}$. Glucagon works to oppose the action of insulin and restore normoglycemia ${ }^{25}$. In response to hypoglycemia, glucagon works by causing the breakdown of glycogen (a multibranched glucose polysaccharide) which is stored in the liver to be released as glucose into the blood stream ${ }^{25}$. 
The rapid rise in glucose concentration after the consumption of a high $\mathrm{Gl}$ meal has been documented in the literature. A study done by Ludwig (2002) showed that within 2 hours of consuming a high GI meal, the blood glucose concentration was twice as much as that found after a low $\mathrm{GI}$ meal ${ }^{26}$. This high glucose concentration from the high $\mathrm{GI}$ meal triggers the increased release of insulin in order to oppose activity from glucagon ${ }^{26}$. Due to the activity of the high levels of insulin, blood glucose levels drop. After 4 hours, almost all the nutrients are absorbed but a high insulin to glucagon ratio can remain ${ }^{27}$. This imbalance in the homeostatic hormones further decreases the blood glucose concentrations. Since blood glucose is the primary source of fuel for the brain, hunger starts to develop ${ }^{6}$. Moreover, the high level of insulin also suppresses lipolysis and thus helps to prevent the use of free fatty acids as fuel ${ }^{26}$. Therefore, a high $\mathrm{Gl}$ food causes the body to undergo similar conditions to fasting.

It is after 4-6 hours of consuming a high $\mathrm{GI}$ meal that low glucose levels start to stimulate the release of glucagon, epinephrine, and cortisol to restore normaglycemia ${ }^{27}$. From the stimulation of these regulatory hormones, cortisol and glucagon, the body attempts to perform glyconeogenesis (synthesis of glucose from non-carbohydrate sources) and glycogenolysis (breakdown of glycogen to glucose), respectively ${ }^{26}$. Epinephrine helps the body to mobilize fat from adipocytes to restore the blood free fatty acid concentrations. However, due to the high levels of insulin, the body's attempts to restore normoglycemia are prevented. It is at this point that hunger intensifies and leads to over consumption of the following meal ${ }^{23}$.

These drastic changes in blood glucose following a high GI meal do not occur with low GI meals. In a low GI meal, the carbohydrates take a longer time to be digested and absorbed which 
results in a gradual change in the blood glucose level ${ }^{23}$. The resulting ratio of insulin to glucagon does not rise dramatically, hence gluconeogenesis, glycogenolysis and lipolysis are not inhibited $^{23}$.

\subsubsection{In vivo measurement of the glycemic index}

In vivo methods are commonly used to determine the Gl after consumption of food. In this test, blood samples are taken from a subject prior to food consumption. After an overnight fast, a portion of food containing $50 \mathrm{~g}$ of available carbohydrates is ingested by a healthy (nondiabetic) participant. Blood samples (venous or capillary) are collected over a $2 \mathrm{~h}$ period at $\mathrm{t}=0$, $15,30,45,60,90$, and 120 min following ingestion and are used to determine blood glucose concentrations. With data from 10 participants, the incremental area under the blood glucose response curves (iAUC) is calculated by the trapezoid rule ${ }^{11}$. Equation 2.1 is used to provide the Gl of the test food.

\subsection{Anatomy and physiology of the gastrointestinal tract}

The gastrointestinal tract (GIT) is a single hollow tube that is $9-10$ meters in length ${ }^{28}$. Its main function is to process and digest the food into a form that can be absorbed into the body via the lymphatic system and the blood stream.

The GIT consists of several compartments: mouth, esophagus, stomach (fundus, body, antrum and pylorus), small intestine (duodenum, jejunum, and ileum) and large intestine (cecum, ascending colon, transverse colon, descending colon, and sigmoid colon and rectum) ${ }^{28}$. Mechanical breakdown in the mouth by chewing and churning action in the stomach helps to break down the digesta into a smaller size and increase the surface area for increased enzymatic 
digestion. This is referred to as the bolus. Peristaltic action helps to move the digesta through the GIT and also assists in mixing. Several accessory glands and organs like the salivary glands, liver, gallbladder and pancreas work with the GIT to help with chemical breakdown of the digesta. Changes in $\mathrm{pH}$ in the different compartments of the GIT help maintain the optimal activity of digestive enzymes ${ }^{29}$.

\subsection{In vivo digestion}

In vivo digestion is a multi-step process involving many organs (Table 2.2). The process of digestion begins in the mouth with the act of chewing food (mastication) which helps to breakdown food and increase surface area for enzymes to act on. Mastication also helps to form food into a bolus (lubricated and salivated portion) by mixing the food with saliva thus helping the food to be swallowed easily. Saliva is produced by serous and mucous acinar cells and secreted mostly by the submandibular salivary glands ${ }^{30}$. Depending on the location saliva is secreted, the magnitude of the salivary $\alpha$-amylase activity changes but is typically $60-70$ $\mathrm{U} / \mathrm{ml}^{31,32}$. Saliva is mostly composed of water, while also containing mucus (which helps to lubricate food and protect the mouth), electrolytes, enzymes such as salivary amylase (which starts the process of carbohydrate digestion) and lysozyme (which protects against bacteria) ${ }^{29}$. Humans typically produce $0.9-1.5 \mathrm{~L}$ of saliva daily ${ }^{29}$. Water in the saliva helps to moisten and soften the food and the mucus helps to bind the bolus thus allowing the food to be easily swallowed.

Food is passed into the esophagus where involuntary contractions push the food into the stomach. Upon reaching the stomach (by passing through the lower oesophageal sphincter), 
salivary amylase becomes inactivated due to the acidic nature of the stomach. This acidic environment brought on by parietal cells that secrete $\mathrm{HCl}$ to lower the $\mathrm{pH}$ is needed for the activation of pepsinogen (secreted by chief cells in the wall of the stomach) to pepsin, a digestive enzyme that aids in breakdown of protein ${ }^{28}$. Coupled with the proteolysis by pepsin, the lower part of the stomach contracts in a rhythmic manner to chum the food inside and mix it with the gastric acid and pepsin (Table 2.2).

The human stomach is a J-shaped organ subdivided into four portions: fundus, body, antrum and pylorus (Figure 2.2). As it is elastic, it can expand to accommodate $1-1.5 \mathrm{~L}$ of food ${ }^{28}$. The stomach contains different types of cells such as chief cells and parietal cells that secrete the needed components to maintain and regulate digestion. Churning, the mechanical process that occurs in the stomach, helps to mix the digesta with gastric juice (composed of $\mathrm{HCl}$, pepsin, mucus and salts) to help with enzymatic digestion. Peristaltic contractions of the antrum help to break down the digesta and push it into the small intestine ${ }^{28}$.

Two types of contractions occur in the stomach: peristaltic and regular tonic. Peristaltic contractions lead to grinding and mechanical breakdown of food ${ }^{33}$ whereas the latter are responsible for moving food from the top to the bottom of the stomach.

The extent of peristaltic contractions is affected by factors such as gender, age, body mass index (BMI) and certain disorders ${ }^{34}$ as well as the physical properties of the food such as fat and solid content ${ }^{34}$. Normally, as the peristaltic waves move toward the pylorus of the stomach, the width of the contractions increase causing the pylorus to contract and the sphincter to narrow ${ }^{33}$. Due to this, only liquids and small particles of the digesta can be driven into the small intestine. 
The larger particles experience retropulsion, where they are pushed back into the stomach for further breakdown ${ }^{33}$.

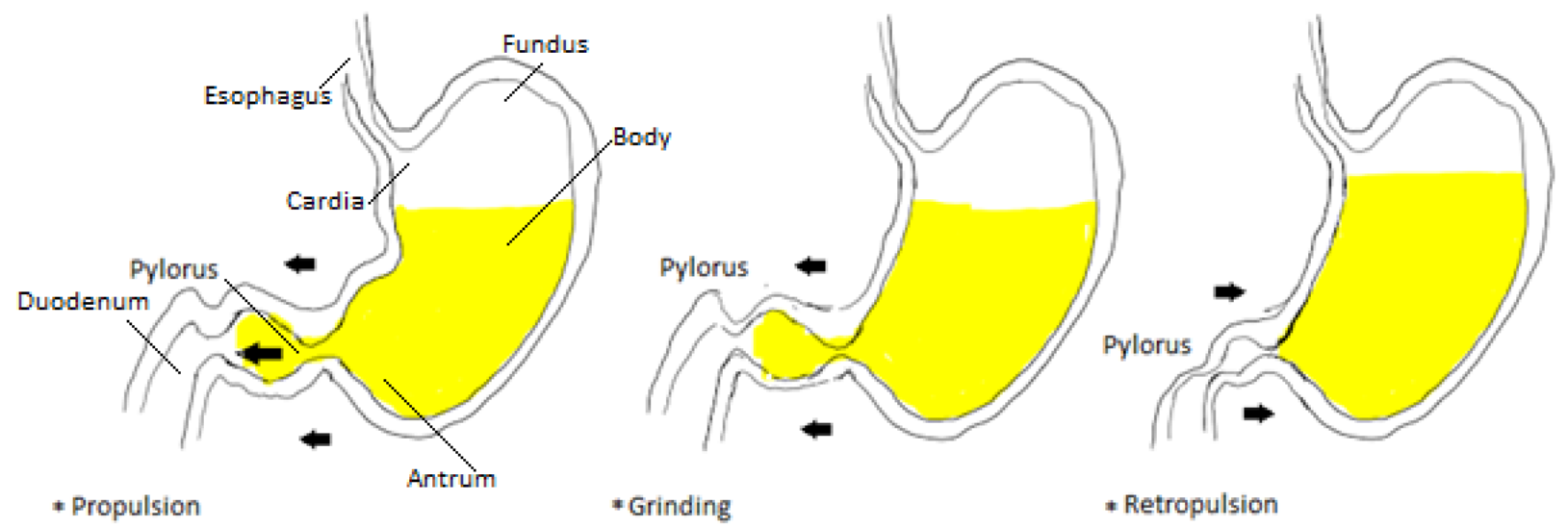

Figure 2.2. Mechanical action from the peristaltic contractions of the stomach. Modified from Kong and Singh, 2008.

Churning and mixing results in the formation of chyme, which is passed into the duodenum (first part of the small intestine) through the pyloric sphincter. After it reaches the duodenum, the chyme is mixed with pancreatic juices that contain bicarbonate (produced by duct cells) that help neutralize it ${ }^{28}$. The majority of food is digested by pancreatic enzymes in the small intestine secreted by acinar cells of the exocrine pancreas ${ }^{35}$. Prior to secretion, the pancreatic juices are mixed with bile secreted by the liver and stored in the gall bladder via bile duct. Bile is a cholesterol derivative made of inorganic ions, bicarbonate, bile salts and phospholipids ${ }^{28}$. Due to its amphiphilic nature, it disperses fats into micelles to increase their surface area which assists in lipolysis by pancreatic lipase ${ }^{28}$. Just as the small intestine is the site where the majority of digestion occurs, it is also the location where most of the absorption of the digested products occurs. The absorption of the resultant products after digestion by the mucosal epithelia is increased due to the mucosal epithelia taking the form of finger like projections 
$\left(\right.$ villii) $^{28}$. During digestion, the three macronutrients, proteins, carbohydrates and fats are broken down into their monomers.

Protein and carbohydrates are broken down into amino acids and monosaccharides respectively and these products are taken up and transported into the blood stream and end up in the liver through the hepatic portal vein. Fats on the other hand are broken down into glycerol and fatty acids and transported as chylomicrons (water-soluble lipoproteins) which help fats and cholesterol to be transported into the lymph and finally into the bloodstream ${ }^{28}$. Absorption across the in mucosal epithelia (in the small intestine) includes processes such as passive and active transport, pinocytosis and even carrier-mediated transport ${ }^{36}$. The last stop for food is the large intestine (colon). This is also the last chance for the body to absorb any water or minerals still remaining. The remaining indigestible content in the large intestine such as fibre is passed on to the rectum where it is expelled out of the body. 
Table 2.2. Enzymes of the gastrointestinal tract. Modified from Verkerstaff Jonegan, 2004.

\begin{tabular}{|c|c|c|c|}
\hline \multicolumn{2}{|c|}{ Site of action } & Enzymes & Action \\
\hline \multicolumn{2}{|l|}{ Mouth } & $\begin{array}{l}\text { Salivary } \alpha- \\
\text { amylase }\end{array}$ & Catalyses the breakdown of $\alpha$-1,4-glycosidic bond \\
\hline \multirow{2}{*}{\multicolumn{2}{|c|}{ Stomach }} & Gastric Lipase & Catalyses the hydrolysis of fats \\
\hline & & Pepsin & Catalyses the cleavage of peptide bonds of protein \\
\hline \multicolumn{4}{|c|}{ Small Intestine } \\
\hline \multirow{7}{*}{ 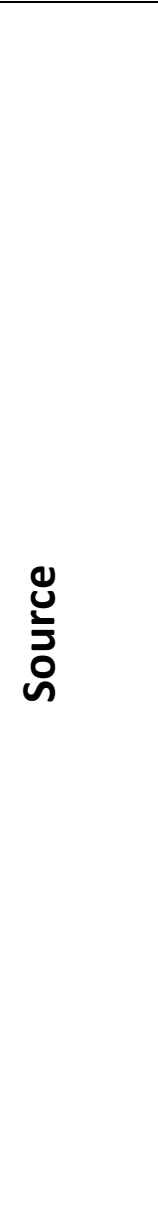 } & \multirow[t]{3}{*}{ Pancreas } & $\alpha$ - amylase & Catalyses the breakdown of $\alpha$-1,4-glycosidic bond \\
\hline & & Proteases & Catalyses the cleavage of peptide bonds of protein \\
\hline & & Trypsin & $\begin{array}{l}\text { Catalyses the cleavage of peptide bonds of protein } \\
\text { specifically for arginine and lysine residues }\end{array}$ \\
\hline & Gall Bladder & Bile Salts & Helps to emulsify lipids \\
\hline & \multirow[t]{3}{*}{$\begin{array}{l}\text { Brush Border } \\
\text { of the small } \\
\text { intestine }\end{array}$} & Lactase & $\begin{array}{l}\text { Catalyses the cleavage of lactose into glucose and } \\
\text { galactose }\end{array}$ \\
\hline & & $\begin{array}{l}\text { Sucrase, } \\
\text { maltase and } \\
\text { isomaltase }\end{array}$ & Catalyses the hydrolysis of glucose and fructose \\
\hline & & $\begin{array}{l}\text { Amino- } \\
\text { oligopeptidases } \\
\text { and dipeptidyl } \\
\text { peptidases }\end{array}$ & Catalyses the cleavage of $\mathrm{N}$-terminal amino acids \\
\hline
\end{tabular}




\subsubsection{Digestion and absorption of carbohydrates}

The majority of the dietary carbohydrates are in the form of starch and the remainder is in the form of oligosaccharides. These polysaccharides must be broken down to their monomers prior to absorption by the body. Starch is made up of amylose (a linear $\alpha-1,4-$ glycosidic linkage polysaccharide) and amylopectin ( $\alpha-1,4-$ glycosidic linkage polysaccharide with $\alpha-1,6$-glycosidic linkage branches $)^{28}$.

The digestion of starch begins in the mouth with the help of salivary $\alpha$-amylase secreted by the serous acinar cells. During the process of mastication, the salivary $\alpha$-amylase is mixed with the bolus and breaks down the $\alpha-1,4$-glycosidic linkage of carbohydrates. As the stomach is acidic, salivary $\alpha$-amylase becomes deactivated and plays a small role in digestion. After the acidic chyme passes into the small intestine, pancreatic amylase further digests the carbohydrates present ${ }^{28}$. The result of the $\alpha-1,4$-glycosidic linkage cleavage in starch (for both amylose and amylopectin) produces maltose and maltotriose (trisaccharide of glucose monomers). However, in amylopectin, $\alpha$-limit dextrins (glucose polymers with $\alpha-1,6$-glycosidic linkage branch points) are also produced. This breakdown of starch happens within ten minutes of the acidic chyme entering the duodenum ${ }^{37}$. These products are further digested by a number of brush border enzymes found on the apical membrane of the small intestine. Thus, maltose is broken down into glucose by maltase. The terminal glucose residues on maltotriose and $\alpha$-limit dextrins are cleaved by glucoamylase ${ }^{28}$. Lactose cleaves lactose into glucose and galactose. Finally, sucrase cleaves sucrose to glucose and fructose. The $\alpha-1,6$-glycosidic linkages of amylopectin are cleaved by isomaltase. Moreover, isomaltase is responsible for cleaving $\alpha$-dextrin to maltose. 
After the digestion of dietary carbohydrates into monosaccharides (glucose, fructose and galactose), they are absorbed. During this process, the body converts fructose and galactose to glucose ${ }^{38}$. Monosaccharide absorption is highly regulated; there are two ways in which they can enter the enterocytes: transcellular or paracellular transport via tight junctions.

Glucose absorption occurs predominantly in the proximal small intestine via sodium glucose cotransporters at the luminal membrane and GLUT2 transporters at the basolateral membrane ${ }^{39}$. Glucose then diffuses into the intestinal villus capillary beds ${ }^{40}$ where it travels to the portal vein, then into the liver after which it is circulated to the entire body via systemic circulation. This is when the glycemic response is generated since glucose is a major source of energy for all tissues.

\subsection{In vitro digestion}

In vivo methods that use human subjects to determine the Gl of food from their glycemic response provide the most accurate results, but they are expensive, highly variable, labourintensive, time-consuming and can make large studies impractical ${ }^{41}$. Therefore, there is a strong case for the development and application of in vitro models that closely mirror the conditions and processes that occur in vivo. Such models have to be sufficiently refined to allow the process of digestion to be followed in some detail and have to be validated against in vivo data ${ }^{42}$. These models are non-invasive, economical and allow for the analysis of large sample sizes ${ }^{42}$. Ideally, an in vitro digestion model should offer the advantages of rapid representative sampling at any time point, and permit testing of whole foods. 


\subsubsection{Advantages of in vitro digestion}

In vitro digestion systems have proven to be powerful tools for understanding and monitoring the complex transformation processes that take place during in vivo digestion ${ }^{43}$. In vitro digestion models provide a useful alternative to animal and human models by rapidly screening food ingredients. There are many benefits to initial screening experiments using these models. They provide significant physicochemical insights into the digestive processes since samples are easier to collect and analyze. They are also more efficient to conduct than animal or human studies in terms of cost and time, allowing for higher turnover of potential delivery systems under study.

A typical in vitro digestion model should consider 4 main stages: (i) mouth (oral phase), (ii) stomach (gastric phase), (iii) duodenum (small intestinal phase) and iv) large intestine (large intestinal phase). These four phases can be considered separately or in combination depending on the purpose of the study. There are two models of in vitro digestion - static and dynamic.

\subsubsection{General background on static and dynamic digestion models}

Static methods are simple to use and usually include 2 or 3 sections of the GIT (oral, gastric, and intestinal). The products of digestion are not removed during the digestion process. This approach may only simulate a limited number of parameters relevant to digestion as physical processes such as shearing and peristalsis may not be mimicked ${ }^{44}$. These models are good for limited digestion but are less applicable for total digestion. These models are used for digestion studies of simple foods or isolated nutrients. Many of these models involve homogenization of the food, then acidification with $\mathrm{HCl}$ with the addition of gastric enzymes followed by a delay to 
simulate gastric residence time, and finally neutralization with sodium hydroxide with the addition of pancreatic enzymes and bile salts all the while stirring at $37^{\circ} \mathrm{C}^{44}$. The progress of the reaction is measured by the rate of loss or appearance of a component ${ }^{44}$. As static models do not have the dynamic environment of the intestine, nutrient absorption does not take place nor are feedback mechanisms and changes in the secretions over time measurable.

A wide array of static models have been used and developed depending on the study design and requirements, which unfortunately has resulted in little consistency. To address this shortcoming, InfoGest, an international group of scientists from different backgrounds studying food digestion have developed a standardized static protocol for simulating digestion in the upper $\mathrm{GIT}^{43}$.

By contrast, dynamic digestion models simulate the continuous changes of the physicochemical conditions that occur during digestion, including changes in $\mathrm{pH}$, peristaltic forces, shear, mixing, hydration and secretion release ${ }^{44}$. Some dynamic digestion models can also simulate nutrient absorption (like the TNO TIM-1).

\subsection{Design of an in vitro digestion model}

From a design perspective, in vitro digestion models can follow three approaches: horizontal, vertical and beaker alignment. Horizontal alignment is good for modeling gastric sieving but carries the disadvantage that it does not offer a good low mixing environment of the fundus. In vitro digestion models based on this alignment include the TIM-1 (TNO GastroIntestinal Model). During digestion, vertical alignment allows for phase separation but due to gravity, sedimentation of larger particles occurs. In vitro digestion models based on this alignment include the Human Gastric Simulator (HGS) and Dynamic Gastric Model (DGM). Some 
models such as the Simulator of Human Intestinal Microbial Ecosystem (SHIME) and in vitro Digestion System (IViDiS) use a beaker alignment, which is similar to static models.

\subsubsection{Current in vitro digestion models}

There have been several different digestion models that have been developed for studying the complex processes of digestion.

\subsubsection{Dynamic Gastric Model (DGM)}

The dynamic gastric model (DGM), developed at the Institute of Food Research (Norwich, UK), is made of two compartments: the stomach and small intestine ${ }^{45}$. The first compartment, the stomach (mainly the fundus), mimics the dynamic conditions that occur in the human stomach like the diffusion profiles of gastric juice as well as gastric emptying. This is based on data from echo-planar magnetic resonance Imaging (EPI) and from human trials ${ }^{46}$. The stomach compartment of the DGM also simulates the antrum and causes the digesta to undergo high shear and mechanical breakdown. The second stage mimics the human small intestine where digesta empties from the stomach and is subjected to simulated intestinal secretions. The DGM was developed to examine the impact of digestion on the bioaccessibility and delivery profiles of nutrients to the duodenum ${ }^{46}$. The DGM also assesses the effects of food structure on nutrient delivery, nutrient interaction and survivability of pharmaceutical drugs ${ }^{46}$.

\subsubsection{Human Gastric Simulator (HGS)}

The Human Gastric Simulator developed by University of California, Davis, is based on the vertical alignment approach. It has a round cylindrical gastric compartment that is squeezed by 
rollers on a latex chamber ${ }^{33}$. The latex body has a diameter of $102 \mathrm{~mm}$ and is able to hold up to 5.7 $\mathrm{L}$ of content. The rollers help to simulate the contractile forces of the antrum. Peristaltic action in the HGS is simulated by having 12 rollers, 4 conveyor belts, and a pulley system ${ }^{33}$. The HGS simulates the actual human stomach contraction cycles by creating three contractions per minute on the latex vessel.

\subsubsection{The TNO Gastro-Intestinal Model (TIM)}

The TNO Gastro-Intestinal Model (TIM) is a multicompartmental model that was developed by TNO in Zeist (the Netherlands) in the 1990s to simulate the lumen of the GI tract. It is a dynamic model that is controlled by a computer to adjust the physiological conditions (such as temperature, peristalsis, secretion of enzymes, flow rates, $\mathrm{pH}$ values) in the $\mathrm{GIT}^{47}$. This model also removes digested compounds and water and has been used to study an array of foods and pharmaceutical products.

The TIM-1 is made up of four compartments, representing the stomach, duodenum, jejunum and ileum ${ }^{48}$. These compartments are connected by peristaltic valve pumps (PVP) that allow the transfer of controlled amounts of chyme from one compartment to another. The PVPs are not easily blocked by large food samples. The temperature is maintained at $37^{\circ} \mathrm{C}$ by controlling the temperature of the water circulating outside the flexible walls. The TIM-1 simulates peristaltic movements, gastric emptying rates and the biochemical changes of digestion in a realistic time-dependent manner ${ }^{48}$. This model has also been used to study the bioaccessibility of food ${ }^{48}$. 


\subsection{Considerations for designing an in vitro digestive tract: Stage by stage}

\subsubsection{Oral stage}

The process of mastication (chewing) in the mouth is influenced by many factors such as the composition and the size of the food, the condition of the teeth, the number of chewing cycles, the force of the bite, and the volume of food ${ }^{49}$. These factors contribute to the size and area of the particle size in the bolus. These differences were observed in a study by Peyron et al. (2004) who compared the boluses of raw vegetables and nuts ${ }^{50}$. They found that the raw vegetables and nuts gave similar boluses made with similar particle sizes to each other ${ }^{50}$. To simulate mastication, the particle size of solid food should be standardized by using a mincer. Versantvoort et al. (2005) suggested that the time of digestion in the oral stage should be $\sim 5$ minutes to accommodate the mechanical process in an in vitro model ${ }^{51}$. However, physiologically when considering the chewing time in vivo, an individual typically chews for a much shorter time. Minekus et al. (2014) suggested 2 minutes for the oral stage of digestion ${ }^{43}$. Although this is longer than the in vivo chewing time, this excess time is needed for the enzymes to be in contact with its substrates and reducing this time may lead to inaccuracies in mimicking the in vivo digestion ${ }^{43}$. Most liquids do not require an oral phase as liquids do not stay very long in the mouth ${ }^{43}$.

Another consideration to be accounted for is the amount of salivary fluid used in the in vitro model. Salivary secretion affects surface tension and viscosity of food in the oral stage ${ }^{31}$. Humans typically produce $1-1.5 \mathrm{~L}$ of saliva per day ${ }^{31}$. Saliva is mainly water but also includes electrolytes such as sodium, phosphate, bicarbonate and calcium. It also contains urea, lysozyme and mucins which are mucosal glycoproteins needed for wetting and lubricating food ${ }^{31}$. For in 
vitro applications, Minekus et al. (2014) suggested that $50 \% \mathrm{w} / \mathrm{v}$ of simulated salivary fluid should be used ${ }^{43}$.

One of the major enzymes in the oral phase is salivary amylase. This enzyme which aids in the digestion of carbohydrates has an optimal pH of $6.8^{52}$. Since digestion of starch happens in the oral stage, this was another argument for the 2 minutes digestion time in the oral stage ${ }^{43}$.

\subsubsection{Gastric stage}

A study by Tyssandier et al. (2003) found that it takes approximately 3 - 4 hours for solid western food (such as hamburgers) to leave the stomach and enter the duodenum. This process is known as gastric emptying ${ }^{53}$. Studies have shown that the gastric emptying of liquid food digestion is very rapid (30 min- $1 \mathrm{~h})^{54}$. As there are many factors that influence the rate of gastric emptying, e.g., the type of nutrients, the changes in $\mathrm{pH}$, etc., it is difficult to mimic the complex digestive processes that occur in the stomach. Taking these factors into account, Minekus et al. (2014) suggested 2 hours is required for gastric digestion.

Dressman et al. (1990) found that after the intake of western food, the $\mathrm{pH}$ of the stomach increased to 5 due to the buffering quality of a western type $\operatorname{diet}^{55}$. However, by secreting $\mathrm{HCl}$, the $\mathrm{pH}$ of the stomach returned to $\sim 2^{56}$. For in vitro tests, Minekus et al. (2014) recommended a $\mathrm{pH}$ of 3 as pepsin works in the rage of $\mathrm{pH} 2-4^{56}$.

\subsubsection{Small intestine}

When the acid chyme from the stomach reaches the small intestine, it is neutralized by bicarbonate and reaches 6.5 in the duodenum and 7.5 in the ileum. These values should be used to simulate the respective components of the small intestine by the addition of simulated 
intestinal fluid and sodium hydroxide. The transit time in the small intestine is affected by many factors but Minekus et al. (2014) recommended a transit time of 2 hours $^{43}$.

There are two main types of secretions, bile and pancreatic enzymes, that are involved in aiding the digestion of macronutrients. Minekus et al. (2014) suggested the use of bile extract or

frozen porcine bile (from porcine gall bladder) and porcine pancreatin respectively ${ }^{43}$. They also recommended porcine trypsin (at a final concentration of $100 \mathrm{U} / \mathrm{ml}$ ) to account for the pancreatic enzymes in an in vitro model. Also, since pancreatin contains many salts, this should also be accounted for by addition of $\mathrm{Ca}^{2+}$ in the simulated intestinal fluid. A concentration of $10 \mathrm{mM}$ of porcine bile was also recommended ${ }^{43}$.

\subsection{Current in vitro methods for carbohydrate digestion}

\subsubsection{Oral phase}

The oral phase involves the mechanical and chemical digestion of starch by $\alpha$-amylase and bolus formation with the help of saliva before swallowing. Some current models opt out of simulating the oral phase in vitro by having human subjects. A study by Akerberg et al. (2010) measured the major forms of resistant starch in food by using several volunteers who brushed their teeth to chew $1.0 \mathrm{~g}$ of total starch for 15 times for around 15 minutes ${ }^{57}$. By having volunteers chew the food prior to further digestion, it exposes the food to salivary $\alpha$-amylase.

Other models have made use of mincers, sieves and food processors to simulate chewing. These methods do not correctly simulate the oral phase since grinding or homogenizing does not produce food particle sizes similar to chewing. Since these processes only mimic chewing, 
Brighenti et al. (1993) incubated the food with human salivary $\alpha$-amylase to introduce salivary enzymatic digestion ${ }^{8}$.

\subsubsection{Gastric phase}

In vivo digestion in the gastric phase involves the digestion of the protein fraction of food by enzymatic hydrolysis by pepsin. Also, the low acidic condition of the stomach allows for further denaturation of food. Gastric emptying of the acidic chyme into the duodenum is affected by factors such as viscosity and the quantity of food.

To mimic these conditions, in vitro methods involving starch digestion started to include the gastric phase with pepsin proteolysis as a way to closely simulate the physiological conditions and also to disrupt protein-starch interactions that may have occurred ${ }^{58}$.

Woolnough et al. (2008) examined the digestion of wheat by including a gastric phase with pepsin ${ }^{59}$. The gastric residence time ranged from 30 to 60 minutes and the $\mathrm{pH}$ was between 1.5 and 2.0. The temperature was held constant at $37^{\circ} \mathrm{C}$. Holm et al. (2006) found that without inclusion of the gastric phase in starch digestion, there were starch-pepsin interactions that restricted $\alpha$-amylolysis in the small intestine ${ }^{60}$. A study by Englyst (1992), which looked at the determination of nutritionally important starch, did not include the gastric phase. This was rectified in a following study where Englyst included a pepsin step for 30 minutes at $\mathrm{pH}$ of $2.0^{22}$. 


\subsubsection{Intestinal phase}

Once the acidic chyme reaches the duodenum from the pyloric antrum, it is mixed with the pancreatic enzymes including $\alpha$-amylase. The enzymes at the brush border of the small intestine also help in the complete hydrolysis of starch into their end product monosaccharides.

In vitro digestion models for the small intestine subject the acidic chyme to pancreatic enzyme secretion that is reflective of what is seen in vivo. Also, a buffer is added to increase the $\mathrm{pH}$ of the solution. However, depending on the type of study being performed, samples can be taken during the digestion process, or at the end or not even taken at all for studies involving the removal of digestible carbohydrate for resistant starch isolation.

Englyst et al. (1992) used a screw top tube system containing pancreatic enzymes, amyloglucosidase and invertase to determine the digestibility of $\operatorname{starch}^{22}$. The hydrolysis proceeded at $\mathrm{pH} 5.3$ for over 2 hours and $0.5 \mathrm{ml}$ aliquots of samples were drawn at 20 and 120 minutes. These two time points were taken because this allowed the authors to describe the portion of digestible starch based on rate of digestion ${ }^{22}$. Englyst et al. (1992) found that rapidly digestible starch was digested within 20 minutes, slowly digestible starch was digested between 20 and 120 minutes and resistant starch remained after 120 minutes of digestion. In another study, Woolnough et al. (2008) used pancreatin at pH 6 and continued the hydrolysis for two hour ${ }^{59}$. Areas under the hydrolysis curve were taken and the GI value was determined by comparing the hydrolysis index to the total starch hydrolyzed at the various time points. 


\subsection{YSI Biochemical Analyzer}

The use of traditional methods such as HPLC (high performance liquid chromatography) and GC (gas chromatography) requires a fair level of expertise to operate, high maintenance and a fairly long time for analysis. The YSI biochemical analyzer provides for real-time, accurate analysis of key food components for various analytes such as many monosaccharides ${ }^{61}$.

This system uses an enzymatic membrane specific to the analytes of interest and measures changes in current across a membrane. An enzyme specific for the substrate of interest (for example, glucose) is immobilized between two membrane layers ${ }^{61}$. The substrate is oxidized as it enters the enzyme layer, producing hydrogen peroxide, which passes through cellulose acetate to a platinum electrode, where the hydrogen peroxide is oxidized ${ }^{61}$. The resulting current is proportional to the concentration of the substrate, e.g., glucose. YSI membranes contain three layers - a porous poly-carbonate which limits the diffusion of the substrate into the second enzyme layer, preventing the reaction from becoming enzyme-limited ${ }^{61}$. The third layer, cellulose acetate, permits only small molecules such as hydrogen peroxide to reach the electrode, eliminating many electrochemically-active compounds that could interfere with the measurement ${ }^{61}$. 


\section{Chapter 3 - Methods and Materials}

\subsection{Materials}

A spectrum of test foods within their expiration date along with a standard food (white bread) was used for in vitro digestion and for the determination of GI. The in vitro digestion of these test products was performed by using $50 \mathrm{~g}$ of available carbohydrates of that product. Each experiment was performed in triplicate. The in vitro digestion process was divided into three main phases: oral, gastric and intestinal phase.

\subsubsection{Step 1: Preparation for in vitro digestion}

Prior to in vitro digestion in the DRUID, a spectrum of carbohydrate-rich foods was selected. These test foods were selected on the basis of their physical and structural properties as well as to reflect a range of GI values from low to high, namely skim milk, red kidney beans, dried white kidney beans, Arrowroot cookies, Golden Delicious apple, Raisin Bran cereal, frozen sweet corn, Quaker Oats, banana, baked French fries, whole wheat bread and white rice. The standard food, white Wonder bread was used as a reference for determining the in vitro Gl of the test food.

Sample preparation:

Preparations were made for the following test food prior to in vitro digestion:

- Dried white kidney beans: boiled for $60 \mathrm{~min}$ in an excess of water after an overnight soak (at room temperature).

- Baked French Fries: baked for 18 minutes at $230^{\circ} \mathrm{C}$ in the oven according to manufacturer's instructions 
- Sweet corn: heated in the microwave for 2 minutes

- White rice: cooked in a rice cooker for 20 minutes

\subsubsection{Preparation for in vitro digestion with an addition of fat}

An additional experiment was performed to examine the dose response of adding butter during cooking on the GI of white rice. In this study, three different amounts of butter (20 g, 40 $\mathrm{g}$ and $80 \mathrm{~g}$ ) were added to white rice ( $50 \mathrm{~g}$ of available carbohydrate portion). The rice was cooked in a rice cooker for 20 minutes before being subjected to digestion in the DRUID.

\subsubsection{Determination of glucose, fructose, sucrose concentration and starch content}

For foods without a nutritional label, the $50 \mathrm{~g}$ of available carbohydrate was calculated by performing glucose, fructose, and sucrose concentration determination assays. Sucrose, fructose and glucose concentrations were determined by following the procedure given by the Sucrose/DFructose/D-Glucose Assay Kit (Megazyme, Bray, Ireland). Using the kit, six different solutions were prepared (labelled from solution 1 - 6). A homogenizer was used to homogenize the solid sample. A $10 \mathrm{~g}$ sample from the homogenate was treated to potassium hexacyanoferrate, zinc sulfate and sodium hydroxide to clarify the sample. A filter was used to filter and obtain a clear, non-pigmented solution from the homogenate. A blank, standard, and the sample were prepared by adding the prescribed amount of the six prepared solutions and water as instructed by the assay's protocol. A spectrophotometer was used to read the absorbance of the sample at 340 $\mathrm{nm}$. A dilution calculation along with Beer's Law was used to determine the concentration of glucose, fructose, and sucrose from the absorbance of the sample. 
The starch content was also determined by following the procedure given by Megazyme. Using the kit, six different solutions were prepared (labelled from solution 1 - 6). The food sample was passed through a $0.5 \mathrm{~mm}$ screen and $100 \mathrm{mg}$ was added to a test tube. To this $0.2 \mathrm{ml}$ of ethanol $(80 \% \mathrm{v} / \mathrm{v})$ was added to wet the sample and the test tube was shaken using a vortex. 1.0 $\mathrm{ml}$ of bottle 1 (containing $\alpha$-amylase) was diluted into $100 \mathrm{mM}$ sodium acetate buffer and the solution was added into the test tube with the sample. The test tube was put into a $100^{\circ} \mathrm{C}$ water bath for six minutes and mixed every two minutes during incubation. $0.1 \mathrm{ml}$ of bottle 2 (amyloglucosidase) was added to the test tube and the test tube was mixed and incubated for 30 $\min$ at $50^{\circ} \mathrm{C}$. The contents of the test tube were transferred into a $100 \mathrm{ml}$ volumetric flask and distilled water was added to reach the $10 \mathrm{ml}$ mark. The flask was mixed well and $1.0 \mathrm{ml}$ of the contents from the flask was extracted and diluted with $10 \mathrm{ml}$ of distilled water prior to centrifugation at 3,000 rpm for $10 \mathrm{~min}$. Aliquots of $0.1 \mathrm{ml}$ of the clear centrifuged filtrate were added to test tubes. $3.0 \mathrm{ml}$ of GOPOD (glucose oxidase/peroxidase) reagent was added to each tube including the glucose control tube and blank. A blank, standard and the sample was prepared by adding the prescribed amount of the six prepared solutions and water as instructed by the assay protocol. A spectrophotometer was used to read the absorbance of the sample at $510 \mathrm{~nm}$. A dilution calculation along with Beer's Law was used to determine the starch content from the absorbance of the sample.

\subsubsection{Step 2: In vitro digestion in the DRUID}

A static in vitro digestion model, Dedicated Ryerson University In-vitro Digester (DRUID) was designed to simulate both the in vivo gastric and intestinal conditions in a controlled in vitro setting. 
The DRUID is composed of two $4.0 \mathrm{~L}$ bioreactors (representing the stomach and small intestine (specifically the duodenum)) that are connected by peristaltic pumps (BRL C600). An overhead stirrer (Bellco Glass Overhead Stirrer) was used to drive the impeller to simulate mechanical breakdown of the digesta as well as to evenly distribute the simulated secretions.

While there are some in vitro GI methods used to determine the GI of foods, most do not employ methods of in vivo testing such as oral, gastric and intestinal digestion and sampling over a $2 \mathrm{~h}$ period in the small intestinal phase. The DRUID simulates both gastric and intestinal conditions to determine the glycemic response of commonly consumed carbohydrate-containing foods.

In the first phase (oral phase), solid carbohydrate-containing foods were mechanically broken down using a Ninja blender (a mechanical blender). Particles smaller than $4 \mathrm{~mm}$ were collected to make up $50 \mathrm{~g}$ of carbohydrates of the food. These food particles were subjected to simulated saliva which was prepared with $\mathrm{NaCl}(150 \mathrm{mM}), \mathrm{KCl}(2 \mathrm{mM}), \mathrm{NaHCO} 3(25 \mathrm{mM})$ and 160 $\mathrm{U} / \mathrm{ml}$ salivary alpha amylase for two minutes.

After the oral phase, the food bolus was driven into the gastric phase of the DRUID (a bioreactor) using a peristaltic pump. Eight $\mathrm{ml}$ of $1 \mathrm{M} \mathrm{HCl}$ were added into the gastric bioreactor. The bolus was then subjected to $10 \mathrm{ml}$ of $10 \%$ pepsin/0.05 M solution (Pepsin EC 3.4.23. from porcine stomach mucosa, Sigma-Aldrich P 7000; 800-2 $500 \mathrm{U} / \mathrm{mL}$ ) along with $300 \mathrm{ml}$ of Milli-Q water. The food was digested in the gastric phase at $37^{\circ} \mathrm{C} 30$ minutes with constant mixing at 150 $\mathrm{rpm}$ (via impeller rotation). The $\mathrm{pH}$ was adjusted to a range of $\mathrm{pH} 1.5-1.75$ using $1 \mathrm{M} \mathrm{HCl}$. One (1) $\mathrm{ml}$ aliquots were taken every 10 minutes (near the impeller, from the top, middle and bottom) 
for further analysis. The samples were neutralized with $60 \mu \mathrm{L}$ of $6 \mathrm{M} \mathrm{NaOH}$. To each sample collected, $4 \mathrm{ml}$ of absolute ethanol was added to stop enzymatic digestion.

In the last step of in vitro digestion in the DRUID, the acidic chyme from the stomach was driven into the intestinal phase bioreactor where it was mixed with $2 \mathrm{ml}$ of $\mathrm{NaOH}$ and $2 \mathrm{ml}$ of amyloglucosidase (EC 3.2.1.3. from Aspergillus niger, Megazyme, E-AMGDF; 3260 U/mL). Also, $100 \mathrm{ml}$ of $2.5 \%$ pancreatin/ $0.1 \mathrm{M}$ sodium maleate buffer solution, $5 \mathrm{mg} / \mathrm{ml}$ bile extract and 10 $\mathrm{mg} / \mathrm{ml}$ of lactase ( $\beta$-Galactosidase from Aspergillus oryzae, Sigma-Aldrich, EC 3.2.1.23) were added. The food was digested in the intestinal phase for 120 minutes at $37^{\circ} \mathrm{C}$ with constant mixing at $150 \mathrm{rpm}$. The $\mathrm{pH}$ was adjusted to a range of $\mathrm{pH} 6.85-7.15$ using $1 \mathrm{M} \mathrm{NaHCO}_{3}$. Samples of $1 \mathrm{ml}$ were taken every 15 minutes starting from $\mathrm{t}=0$ minute. Enzymatic activity was deactivated with the addition of absolute ethanol and samples were centrifuged prior to determination of glucose concentration. The volume was maintained at $500 \mathrm{ml}$ with Milli-Q water. Samples were stored at $4{ }^{\circ} \mathrm{C}$ until further analysis.

All trials were performed in triplicates and all secretions were prepared fresh right before the start of each trial.

\subsubsection{Step 3: Analysis of glucose concentration}

A YSI 2700 biochemical analyzer (YSI, Ohio, USA) was used to determine the glucose concentration of the foods after digestion in the DRUID. A $25 \mu \mathrm{L}$ aliquot of the sample was aspirated by the YSI 2700 for determination of the glucose concentration. 
Alongside this, a commonly used colorimetric assay from Megazyme (GOPOD; glucose oxidase/peroxidase kit) was used to determine the glucose concentration of the samples after digestion in the DRUID as a way of validating the YSI 2700 . The GOPOD assay was performed in the dark where $0.1 \mathrm{ml}$ of sample was mixed with $3.0 \mathrm{ml}$ of GOPOD reagent and incubated at $50^{\circ} \mathrm{C}$ for 20 minutes. The absorbance values were read at $510 \mathrm{~nm}$ and a UV/VIS spectrometer was used to calculate the concentration of glucose for the samples collected after digestion in the DRUID.

The glucose concentrations over the duration of the digestion process for each test and standard food were used to generate a graph of the concentration of glucose during digestion. The in vitro GI value of each solid test food was determined by calculating the incremental area under the curve (iAUC) of the test food with respect to white bread. The incremental area under the curve was calculated using the trapezoid method as proposed by Wolever $(2004)^{62}$ and compared to published in vivo GI values ${ }^{63}$.

\subsection{Data analysis}

All results reported in this thesis are the arithmetic mean \pm standard deviation of triplicate experiments. GraphPad Prism 6 and Microsoft Excel 2016 were used to generate the glucose response curves and for calculating the GI of food. Statistical analysis was performed using SPSS v 23.0 (SPSS Inc., Chicago, IL). A Bland-Altman plot was used to assess the agreement between published in vivo and in vitro GI values. Pearson's correlation was used to assess the strength of the relationship of published in vivo and in vitro Gl values. 


\section{Chapter 4-Results and discussion}

\subsection{Introduction}

Due to the lack of a standardized method for the in vitro determination of $\mathrm{Gl}$, sample preparation is different in each in vitro digestion method ${ }^{22,24,33}$. The sample may be broken down by mincing or by using subjects to chew the samples. In vivo methods for determination of $\mathrm{Gl}$ employ volunteers to chew food samples, however some criticize chewing as it varies from person to person as the composition of the saliva is different from person to person ${ }^{86}$, which may contribute, in part, to some of the variability of the GI. Recent studies have shown that mincing technique showed good potential for mimicking the oral digestion process for carbohydrate containing food ${ }^{33}$. To maintain similar particle sizes in the food samples, this study used a Ninja Blender for mechanical digestion in the oral phase of the DRUID for collecting food particles below $4 \mathrm{~mm}$ in size.

The test foods selected for this study were chosen on the basis of their physical and structural properties as well as their typical consumption within the human diet. They range from solids to semi-solids and liquids and have a range of in vivo GI values from low to high. White bread was used as a reference for determination of the in vitro Gl of the test food. 


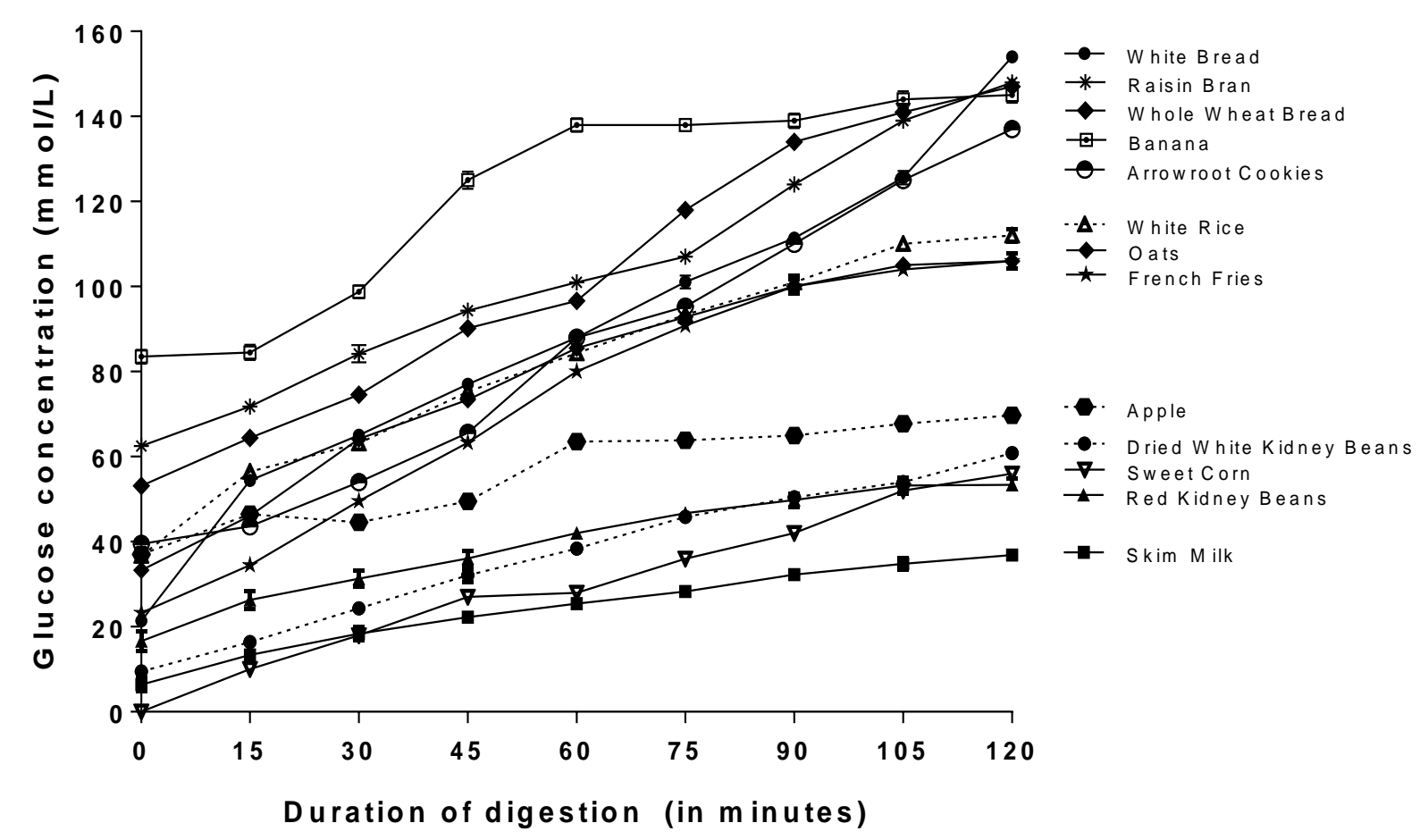

Figure 4.1. Breakdown of food against white bread (50 g of available carbohydrates) in the small intestinal compartment of the DRUID.

Figure 4.1. shows the change in glucose concentration of the test food over $2 \mathrm{~h}$ in the intestinal phase of the DRUID. The glucose concentrations of all foods increased over time, with skim milk yielding the lowest concentrations of glucose release. 


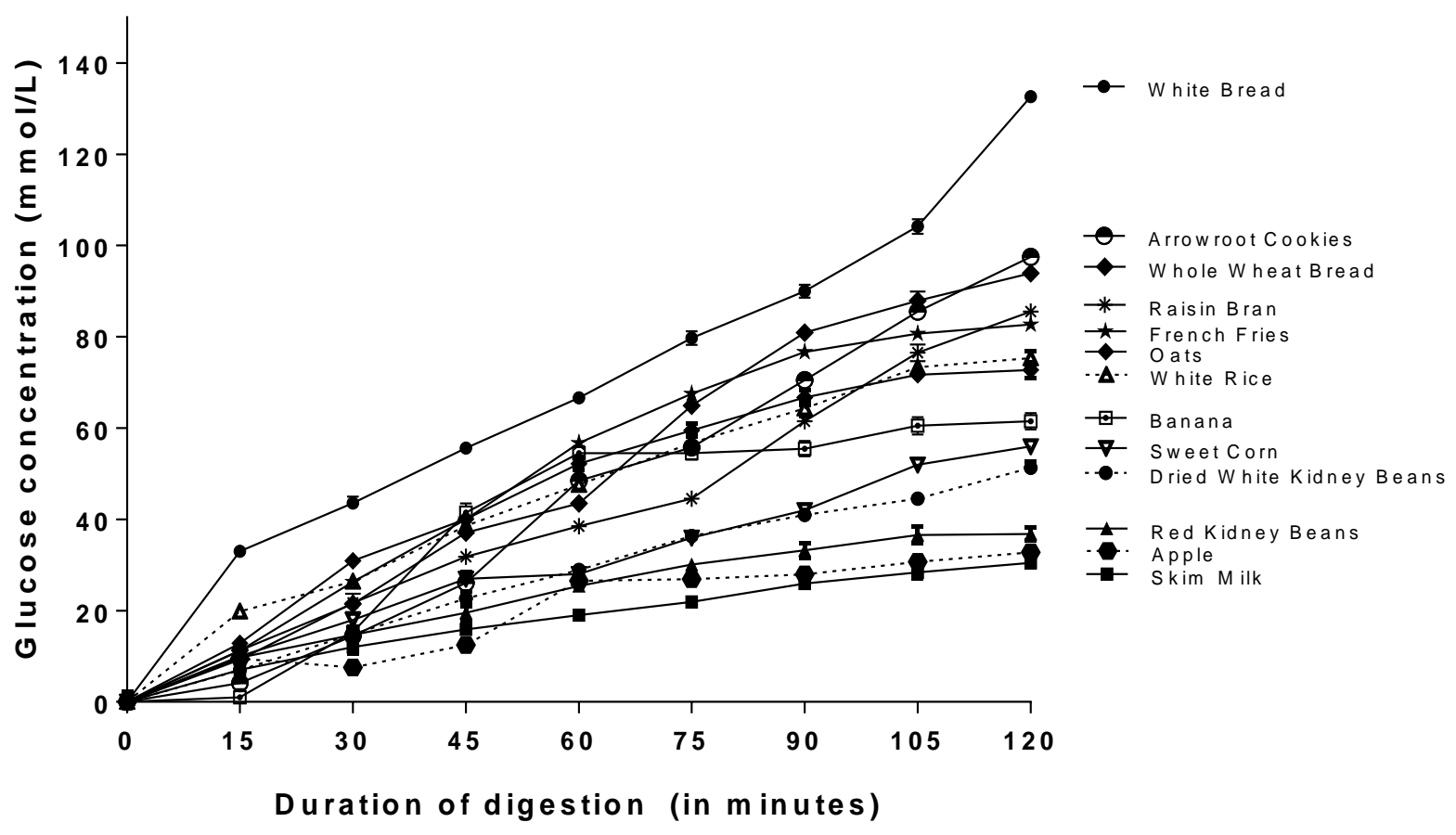

Figure 4.2. Change in glucose concentration from baseline of the test food compared with the control over $2 \mathrm{~h}$ in the small intestine compartment of the DRUID.

White bread had a lower initial glucose concentration when compared with many of the test foods, though not the beans, sweet corn and skim milk. This was expected as it consisted of little free glucose prior to digestion (4\%). The increase in glucose concentration during intestinal digestion occurred as the starch in the bread was hydrolyzed to glucose due to the action of pancreatic amylase cleaving the $\alpha-1,4$-glycosidic linkage ${ }^{38}$. Maltase then cleaves the disaccharide maltose (two glucose units) and isomaltase cleaves $\alpha-1,6$-glycosidic linkage ${ }^{38}$. Given that the DRUID is an in vitro digestion model that does not simulate nutrient absorption, the resultant available glucose arising from the free glucose component of each food and the glucose release after carbohydrate digestion accumulates over the $2 \mathrm{~h}$ residence time in the small intestinal compartment of the DRUID. When glucose release was compared between all the selected foods 
(Figure 4.2), white bread had the highest increase in glucose concentration and skim milk the lowest.

Skim milk expectedly had the lowest initial glucose concentration of all test foods given that most of its carbohydrates are in the form of lactose ${ }^{64}$. Also, it was expected that the glucose concentration of the skim milk would be constant in the stomach since there are no carbohydrate-digesting enzymes. Due to the low $\mathrm{pH}$ of the stomach, it was expected that the low $\mathrm{pH}$ would cause the casein present in milk to coagulate leading to slow gastric emptying thus reducing the rate of lactose digestion and thereby lowering the GI value ${ }^{64}$.

When the acidified skim milk was transferred into the small intestine, pancreatin and lactase was added. Due to the presence of lactase, it was expected that the conversion of lactose to glucose and galactose would occur. This was found to be the case as in Figures 4.1 and 4.2., it can be seen that there was a slow increase in the glucose concentration over time for the skim milk. This was as expected since the carbohydrates of milk are composed of lactose and in the small intestine (specifically in the brush border region), the enzyme lactase would cleave the lactose up into glucose and galactose. Although, in the body this process is a slow one.

During the gastric phase, there was a constant glucose concentration present for all of the test food since carbohydrates were not digested due to the lack of digestive enzymes for carbohydrates. Treatment in the gastric phase may have allowed for the disruption of the protein-starch interactions present in the test food and thus helped to increase accessibility of enzymes to carbohydrates for digestion ${ }^{58}$. Arrowroot cookies, French fries, whole wheat bread, and banana were expected to have the highest GI on the basis of their granular structure (due to 
the soft amorphous layers of their starch granules) ${ }^{65}$ as observed in Figures 4.1 and 4.2. Moreover, Arrowroot cookies and banana had one of the highest initial concentrations of sugars ( $30.8 \%$ and $15.1 \%$ respectively). Both baked French fries and white rice were subjected to heating prior to in vitro Gl determination. The high temperatures these foods were subjected to cause the starches to experience a loss in granular structure due to weakened hydrogen bonds from temperatures of $60-70{ }^{\circ} C^{65}$. This causes the starch granules of these foods to take up water and swell in a process known as gelatinization and thus become more susceptible to hydrolysis by $\alpha$ - amylase ${ }^{65,66}$. As a result, heating of these foods caused an increased glucose release and thus resulted in a relatively high GI value (Figures 4.1 and 4.2). Conversely, the legumes, sweet corn and apple were expected to have a lower release of glucose given their fibre content which hindered enzymatic digestion. It was found that although the macronutrient composition of dried white kidney beans and red kidney beans were similar, dried white kidney beans had an in vitro $\mathrm{Gl}$ of $41.0 \pm 0.5$ whereas the red kidney beans had an in in vitro $\mathrm{Gl}$ of $32.1 \pm 0.8$. These GI values were very similar to what was found in vivo ${ }^{63}$. The reason for this difference in GI arose due to the preparation of the dried white kidney beans. Prior to digestion and GI determination, the dried white kidney beans were subjected to $60 \mathrm{~min}$ of boiling. Boiling is a factor that affects GI by increasing gelatinization and starch digestibility and thus results in an increased glucose response ${ }^{67,68}$.

Table 4.1. shows the in vitro and published in vivo GI values for the selected foods. The Gl of many foods worldwide have been tested in vivo, and most have been incorporated into the "International tables of glycemic index and glycemic load values: 2008 "69. The in vivo GI values used in Table 4.1 were calculated after using glucose as the standard food ${ }^{63}$. The reason for this 
was because unlike when glucose was used as the standard food to calculate the in vivo GI, many of the in vivo Gl values of food products from an article by Foster-Powell et al. (2002) underwent $3 \mathrm{~h}$ of intestinal digestion when white bread was used as the standard food ${ }^{63}$. When glucose was used as the standard food to calculate the in vivo $\mathrm{Gl}$ of food products, the intestinal trials were performed for $2 \mathrm{~h}$ (similar to how other in vivo $\mathrm{Gl}$ trials were conducted as well as how $\mathrm{Gl}$ determination was conducted in the DRUID) ${ }^{63}$. Moreover, many papers examining in vivo GI only reported in vivo GI values of foods when glucose was used as the standard food. Furthermore, when the $\mathrm{Gl}$ of food was calculated under white bread (in the article by Foster-Powell et al. (2002)), some food had Gl over 100 meaning that these values were over the glycemic index scale $^{63}$. This was not observed with the in vivo Gl values calculated when glucose was used as the standard food.

For the determination of GI in vitro, white bread was used as the standard food (Table 4.1). The reason for this was because when white bread was digested in the DRUID, there was an accumulation of glucose concentration over the digestion period since the DRUID does not simulate nutrient absorption and this yielded a high iAUC. This was also what was found for the test foods (Figures 4.1 and 4.2). However, when glucose was digested, there was only a very small change in the glucose concentration over the digestion period since glucose solution already exists in its monomeric form of glucose units. As a result, when glucose was used as the standard for the test foods, the GI was over 100 since the iAUC for glucose was relatively very small when compared to the iAUC of the test foods. As such, white bread, having the highest iAUC of all the test foods was used as the standard for in vitro determination of GI. 
In Table 4.1, unlike the in vitro GI values, the in vivo GI values have relatively high standard deviations or foods were not tested in duplicate. This shows that the digestion in the DRUID can be more reproducible as the determined in vitro GI does not vary significantly from trial to trial. From looking at Table 4.1., it can be seen that for nearly all of the test food, the in vitro GI values determined after the digestion in the DRUID were similar to what is found from published in vivo GI values. Moreover, a Bland- Alman plot, which is typically used to analyze the agreement between two types of measurements was used to analyze the agreement between the in vitro and in vivo $\mathrm{Gl}$ values as seen by Figure 4.3. The Bland-Altman plot showed that there was agreement between the GI values determined from the DRUID when compared with published in vivo Gl values. From Figure 4.3, it can be seen that all of the foods are clustered around the line of no difference. 
Table 4.1. Comparison of experimental in vitro and in vivo GI values for a series of foods. In vivo values are from a previously-published report ${ }^{63}$.

\begin{tabular}{|c|c|c|c|}
\hline Food item & $\begin{array}{l}\text { in vitro GI } \\
\text { values }\end{array}$ & $\begin{array}{l}\text { in vivo } \mathrm{GI} \\
\text { values }^{63}\end{array}$ & $\begin{array}{c}\% \text { difference between } \\
\text { the GI values }\end{array}$ \\
\hline White Bread & 100.0 & 100.0 & $0.0 \%$ \\
\hline French Fries & $74.3 \pm 0.4$ & $75.0 \pm n d$ & $-1.0 \%$ \\
\hline Whole Wheat Bread & $73.1 \pm 0.4$ & $71.0 \pm n d$ & $+3.0 \%$ \\
\hline Arrowroot Cookies & $70.3 \pm 0.4$ & $63.0 \pm n d$ & $+10.4 \%$ \\
\hline Oats & $68.7 \pm 0.7$ & $65.0 \pm n d$ & $+5.7 \%$ \\
\hline White Rice & $67.6 \pm 0.3$ & $72.0 \pm 9$ & $-6.1 \%$ \\
\hline Raisin Bran Cereal & $60.6 \pm 0.5$ & $61.0 \pm 5.0$ & $-0.7 \%$ \\
\hline Banana & $57.7 \pm 0.9$ & $62.0 \pm 9.0$ & $-6.9 \%$ \\
\hline Sweet Corn & $44.7 \pm 0.8$ & $47.0 \pm n d$ & $-4.9 \%$ \\
\hline Dried White Kidney Beans & $41.0 \pm 0.5$ & $42.0 \pm n d$ & $-2.4 \%$ \\
\hline Red Kidney Beans & $32.1 \pm 0.8$ & $27.0 \pm n d$ & $+16.0 \%$ \\
\hline Apple & $29.4 \pm 0.9$ & $28.0 \pm n d$ & $+4.9 \%$ \\
\hline Skim Milk & $27.0 \pm 0.2$ & $32.0 \pm n d$ & $-16.9 \%$ \\
\hline
\end{tabular}




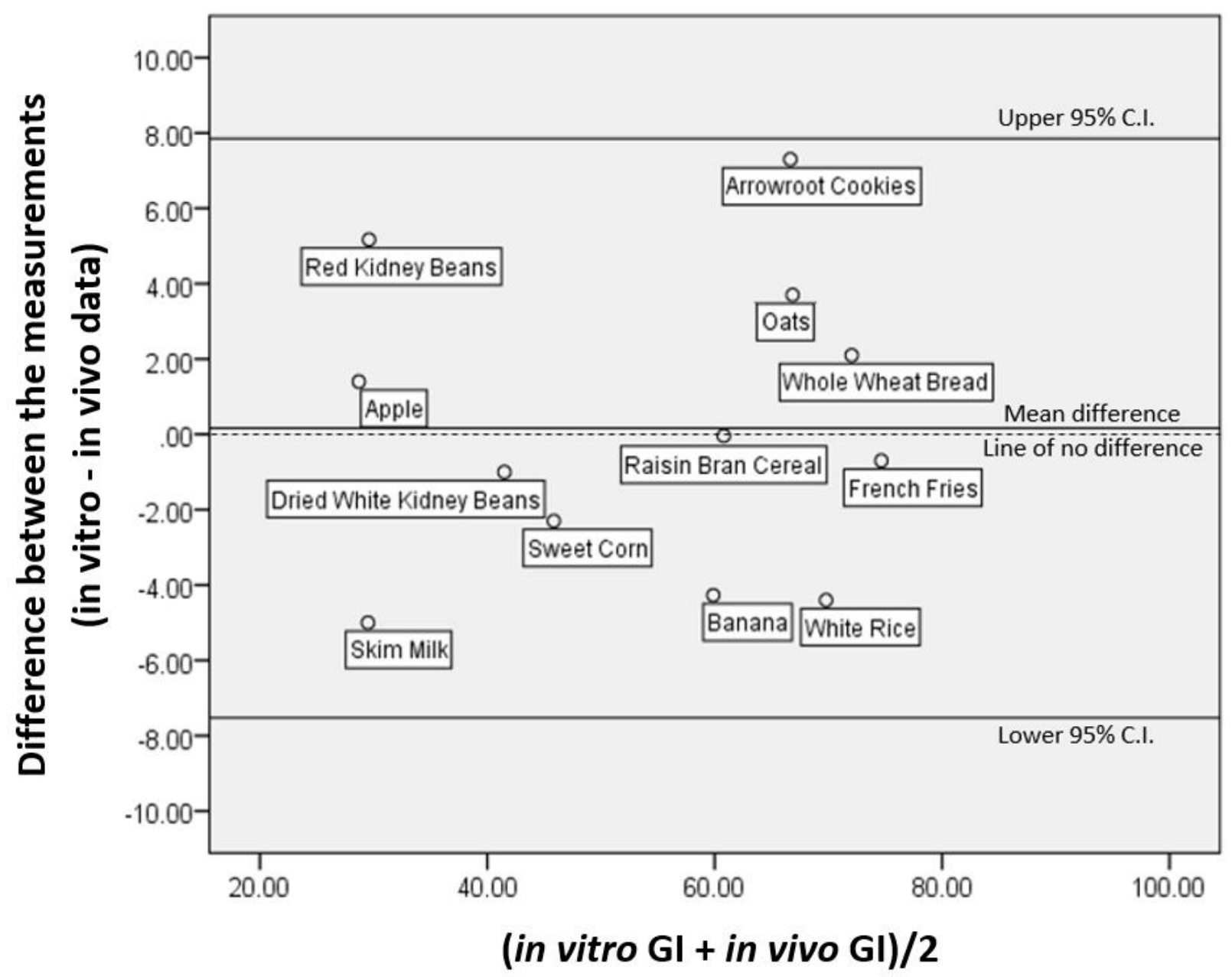

Figure 4.3. Use of a Bland- Altman plot to analyze the agreement between the in vivo and in vitro GI determined values.

Factors including the type of starch, viscosity of fibre, ripeness, food processing, and cooking could have impacted the determination of the in vitro Gl of the red kidney beans and banana. Other factors such as sugar, fat, and protein content can slow down the rate of gastric emptying ${ }^{70}$. Since some foods like the red kidney beans and banana did not have a known procedure for preparing these foods or nutritional labels for Gl testing, factors influencing the determination of $\mathrm{GI}$ values could have yielded a high percent difference between their in vitro and in vivo GI values. These unknown preparation conditions may have affected the proportion 
of amylose to amylopectin, and thus the glucose concentration. Since amylose is more tightly packed when compared to amylopectin, there is a slower rate of digestion as the enzymes have less access to cleave the glycosidic linkages ${ }^{71}$. Also, food processing and cooking can swell up the starch molecules and soften the food which would also speed up the rate of digestion ${ }^{17}$. All of these factors could contribute to a high percent difference between the in vitro and in vivo $\mathrm{Gl}$ values of food if the preparation conditions of GI testing are unknown.

It is therefore important to determine the available carbohydrates of these foods prior to determining the in vitro or in vivo Gl. For a majority of the test foods, nutritional labels were supplied and from this the $50 \mathrm{~g}$ of available carbohydrate was calculated. For raw foods like banana which did not have a nutritional label, assay kits were used to determine the concentration of glucose, fructose and sucrose as well as the amount of starch prior to in vitro digestion in the DRUID. It was found that in $10 \mathrm{~g}$ of a ripe banana, there was $1.50 \pm 1.1 \mathrm{~g}$ of sugar and $2.13 \pm 1.3 \mathrm{~g}$ of starch. Even without the manufacturer's nutrition label for Gl determination, by determining the total carbohydrates present in the ripe banana, the in vitro GI determined after digestion in the DRUID was able to be compared to literature values and thus reducing the percent differences from inter-banana variation and unknown amount of available carbohydrates.

Once the conditions for $\mathrm{Gl}$ determination were known or the available carbohydrates have been determined via assay kits, the DRUID was able to reliably determine the GI of a spectrum of food (as seen by Table 4.1). This shows that, even with the many factors that effect the in vitro GI determination, namely the type of starch, content of fibre, food processing, and 
etc., when given the knowledge of composition and preparation of the food the DRUID was able to accurately determine the $\mathrm{Gl}$ with excellent agreement to published in vivo $\mathrm{Gl}$ values ${ }^{63}$.

The in vivo $\mathrm{Gl}$ values that were selected for the test foods were limited by the available GI literature since no parallel in vivo trials were run with the DRUID. Ideally, in vivo GI values determined after subjecting the test food from Canada to healthy subjects were to be chosen. However, due to the limitation of the available GI literature, the in vivo GI values for baked French fries, Arrowroot cookies, oats, sweet corn, and dried white kidney beans were determined after subjecting the foods to diabetic subjects since no GI values were determined for healthy subjects. This introduces discrepancies between studies as it is the primary investigators that choose what $\mathrm{GI}$ values to report ${ }^{72}$. Further discrepancies may be caused by the use of the limited available $\mathrm{GI}$ literature instead of directly conducting the in vivo GI trials for the test foods. Another limitation of this study was that for the test foods with a given nutritional label from the manufacturer, the nutritional information and available carbohydrates were not measured. Only for the foods with no nutritional information like apple and banana, the available carbohydrate content was measured. Other in vitro methods in previous literature also used the manufacturer's nutritional information of foods in order to estimate their glycemic response $\mathrm{e}^{57,73}$.

\subsection{The DRUID against other in vitro GI determining methods}

Figure 4.4 shows the correlation between the in vitro GI values determined by the DRUID and the published in vivo $\mathrm{GI}$ values for the foods tested ${ }^{63}$. A strong positive correlation between the in vitro GI values determined by the DRUID and the published in vivo GI values, $r=0.9761, p$ $<0.0001$ was observed. This was also evident from the Bland-Altman plot which showed that 
there was an agreement between DRUID determined GI values and the published in vivo GI values.

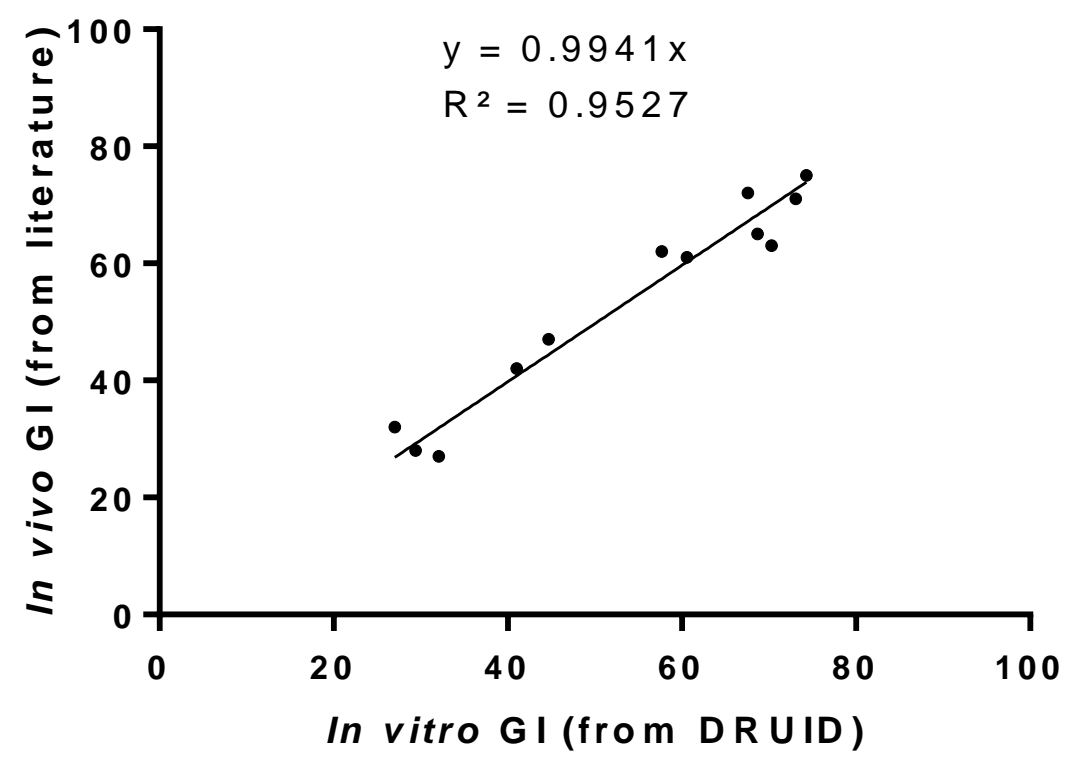

Figure 4.4. Correlation between the in vitro GI values determined by the DRUID and the published in vivo $\mathrm{Gl}$ values for the tested foods ${ }^{63}$.

There are several in vitro methods for the prediction of GI. One of these methods involved incubating the test food with a set of digestive enzymes and afterward performing colorimetric assays to measure the glucose release of test food after 20 min of digestion ${ }^{74}$. The correlation between the in vitro $\mathrm{Gl}$ and the in vivo GI values was reported by Englyst et al. (1996) to be $r=$ 0.58 for a sample of 39 food $^{74}$. This showed that there was a weak correlation between the in vitro $\mathrm{Gl}$ and the in vivo $\mathrm{G}$ / values thus suggesting that predictive validity of this method is low.

Another method developed by Magaletta et al. (2010) involved the enzymatic digestion of $0.5 \mathrm{~g}$ of available carbohydrates of 72 test foods using $\mathrm{HCl}$ and performing $\mathrm{HPLC}$ for the analysis of sugars and sugar alcohols ${ }^{75}$ and the $\mathrm{Gl}$ was assessed by using nonlinear regression modelling . The GI correlated strongly with published in vivo values $\left(r^{2}=0.93\right)$. This method has a strong 
correlation between the predicted GI against the publish GI values for the tested food. This suggests that this method has a good predictive ability for samples outside the sample set. Unlike the DRUID, however, this method used a cryogenic freezer mill for preparing a solid test food for digestion which does not simulate the oral phase of humans. When compared to the DRUID, the use of HPLC for analyzing the different types of sugars and sugar alcohols and the curve fitting of this data using a nonlinear regression model for predicting $\mathrm{Gl}$ is time consuming, requires complex calculations and trained personnel.

A modified digestion method from Argyri et al. (2016) subjected the $0.25 \mathrm{~g}$ of available carbohydrate containing test food to oral, gastric and intestinal phases ${ }^{73}$. Argyri et al. (2016) performed gastric and intestinal digestion of test foods such as breakfast cereals, spaghetti, and banana ${ }^{73}$. The incubation period for the gastric phase lasted for $2 \mathrm{~h}$ while subjected to pepsin and $\mathrm{HCl}$. A dialysis membrane was used for incubating the digestive enzymes for the intestinal phase for $2 \mathrm{~h}$. Unlike the DRUID, dialyzable glucose samples from the intestinal phase were collected every 30 min. Dinitrosalicylic (DNS) assay (a colorimetric method) was used to determine the concentration of dialyzable glucose from the collected samples. The predicted in vitro GI values were compared to published GI values. The correction was nonlinear and Spearman Correlation was used to show the relationship between the predicted in vitro GI values from this method and published GI values. It was found that there was a strong relationship between predicted in vitro $\mathrm{GI}$ and published GI values, Spearman's rho $=0.800, p=0.010$.

Although a few of these methods have predicted in vitro $\mathrm{Gl}$ of test foods that have a relatively strong correlation with the published in vivo GI values, the DRUID, being a fully functional static digestion model with gastric and intestinal compartments that are connected 
via peristaltic pumps was able to yield in vitro GI values that strongly correlated and agreed with the published in vivo GI values $(r=0.9761, p<0.0001)$ (Figures 4.3 and 4.5). Moreover, the DRUID was able to determine the $\mathrm{Gl}$ of a spectrum of foods with high reproducibility and reliability.

\subsection{Effects of the addition of fat on glycemic index of food}

The inclusion of high fat, high protein, or high fibre food in a carbohydrate-rich food may alter the GI. In the human body, proteins can reduce the Gl by stimulating insulin and slowing gastric emptying ${ }^{76}$. Fibre also helps to reduce the $\mathrm{Gl}$ of food by reducing the available carbohydrates for absorption by fibre-glucose binding as well as by slowing the rate of gastric emptying ${ }^{77}$. The consumption of fat with carbohydrates has also been shown to reduce $\mathrm{Gl}$ and blood glucose response ${ }^{78}$. There have been several studies that have shown an impact of fat on GI. Collier and O'Dea (1983) showed that the addition of butter to a potato meal produced a $50 \%$ reduction in glucose response compared to potatoes alone ${ }^{79}$.

The mechanism by which fat reduces the postprandial glycemic response is by delaying the rate of gastric emptying as well as by reducing enzymatic accessibility to carbohydrates 37,48 . Because the DRUID does not simulate gastric emptying and a fixed incubation period was used in the gastric phase, the effect of fat on reducing enzymatic accessibility was determined. ${ }^{51}$.

In order to examine the effect of fat on $\mathrm{Gl}$, a dose response study of dietary fat (20 g, 40 $\mathrm{g}$ and $80 \mathrm{~g}$ of butter) added to white rice was performed. White rice alone had a GI of $67.6 \pm 0.3$ and the addition of the butter dose dependently decreased the glycemic response by $9.0 \%$, $20.6 \%$, and $31.7 \%$ to white rice alone. The $\mathrm{Gl}$ for rice with 20,40 , and $80 \mathrm{~g}$ of butter was $61.5 \pm 1.4$, $53.7 \pm 3.6$, and $46.2 \pm 2.3$, respectively (Figure 4.5 ). The results suggest that cooking rice with butter 
hinders enzymatic digestion and thus helped to reduce the $\mathrm{Gl}$ of the white rice with increasing amount of added butter (Figure 4.6). This reduction in the GI after the addition of fat was also seen in an in vitro study by Crowe et al. where they showed that there was a $35 \%$ reduction in the rate of potato starch digestion with the addition of several fatty acids ${ }^{80}$.

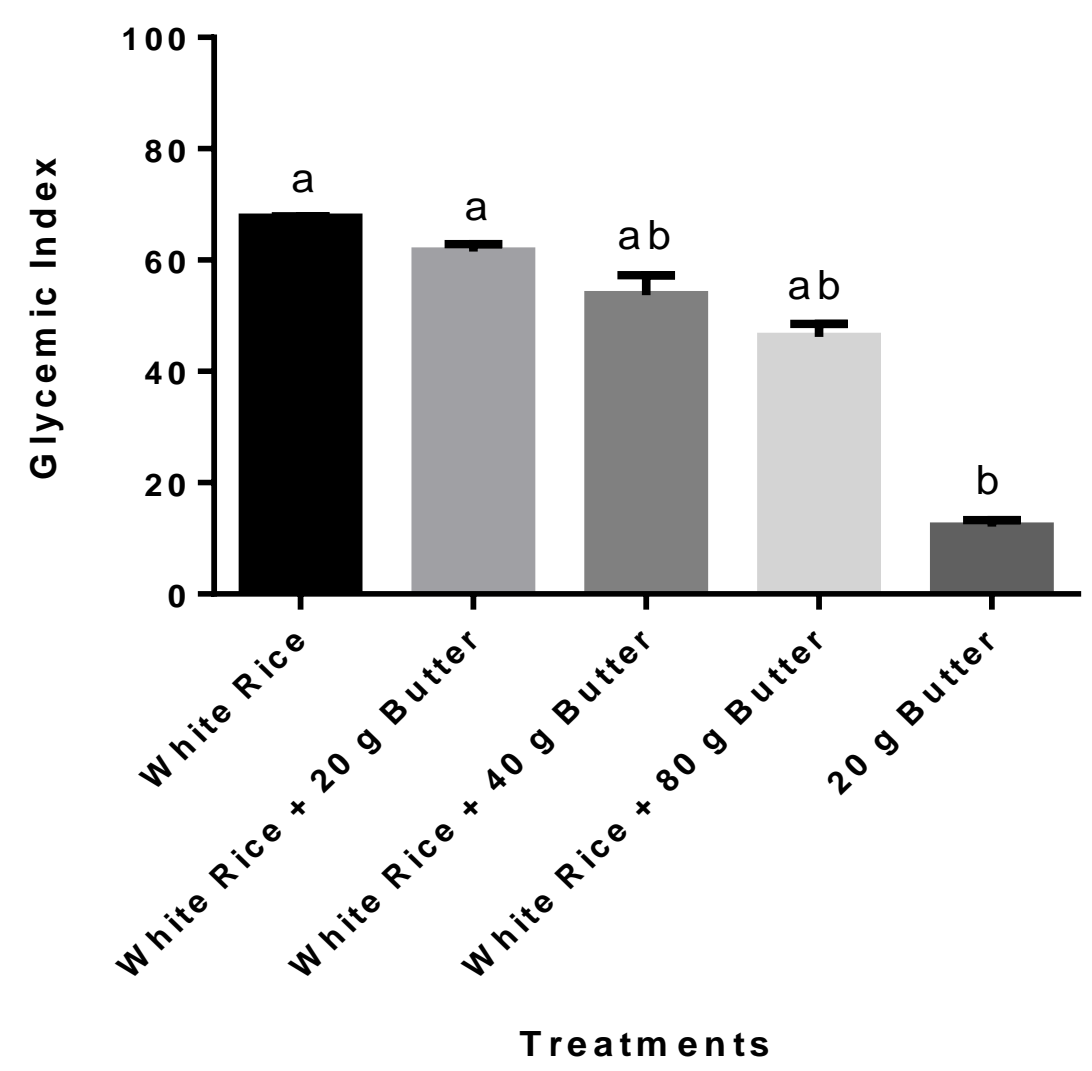

Figure 4.5. The effect of fat on the glycemic index of white rice against white bread. Means with the same letter are not significantly different $(p>0.05)$ 


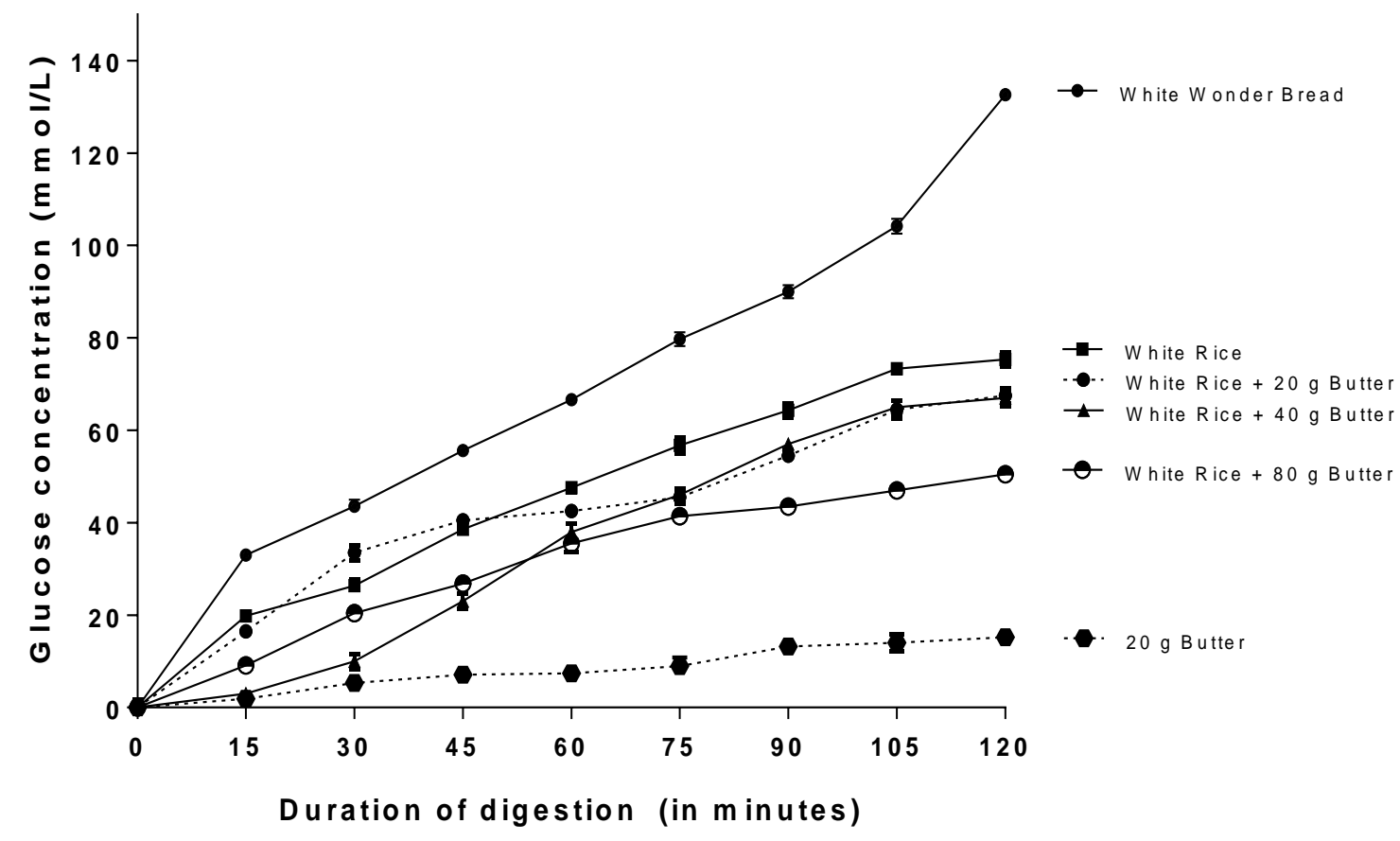

Figure 4.6. The effect of fat on the breakdown of white rice against white bread ( $50 \mathrm{~g}$ of available carbohydrates) in the small intestinal compartment of the DRUID. 


\section{Chapter 5-Conclusions}

The in vitro GI values strongly correlated with published in vivo values and showed a positive relationship between the in vitro and in vivo determined $\mathrm{Gl}(r=0.9761, \mathrm{p}<0.0001)$, and the Bland-Altman plot showed there was a strong agreement between the DRUID determined and published in vivo GI values. Moreover, the DRUID was sensitive enough to detect changes in GI as a result of preparation. Therefore, the DRUID can reliably, cost effectively and reproducibly determine the $\mathrm{Gl}$ across a spectrum of carbohydrate-containing foods, and has the potential to predict the digestion kinetics of novel food products in vivo that may promote human health. 


\section{Chapter 6 - Future studies}

The results from this research highlight the potential of this in vitro GI determining model for upcoming research:

1. In the subject of determining glycemic index, the effect of fiber, protein, processing and, temperature on influencing the GI of food can all be studied by using the DRUID as an in vitro digestion model. This will provide better understanding of the parameters needed to predict the $\mathrm{Gl}$ of food prior to in vivo testing.

2. Parallel in vivo studies for GI determination should also be performed to complement the results from GI determination in the DRUID.

3. Given the determination of GI of enough foods in the DRUID, the in vitro data from the DRUID can be used to create a predictive algorithm for predicting the $\mathrm{GI}$ of food as expected by in vivo GI testing.

4. The DRUID can be used as an in vitro digestion model to determine the GI of mixed meals. This will provide more information on how mixed meals influence the prediction of GI after digestion in an in vitro digestion model. 


\section{Appendix A}

\section{Development of a simple in situ model of the gastrointestinal tract for visualizing the digestion of milk proteins over time via confocal microscopy}

An in situ in vitro model was designed for simulating the gastric and intestinal conditions over time using a confocal microscope. This model was used to observe the physical, chemical and structural changes of protein from skim milk. During in vitro gastric digestion, casein started to coagulate under low acidic conditions. Whey protein was soluble during the in vitro gastric digestion. During in vitro intestinal digestion, most of the casein and residual peptides were hydrolyzed by trypsin and chymotrypsin, and this led to the destabilization and coalescence of the casein micelles. This study brings new ideas on visually observing the digestion of bovine milk in situ.

\section{Introduction}

Milk has been recognized as one of the major sources of protein for people of all ages ${ }^{81}$. Milk protein contains all nine essential amino acids needed by humans ${ }^{81}$. Due to the resemblance to human milk, cow's (bovine) milk is the most abundant source of milk for dairy industries ${ }^{82}$.

Bovine milk consists of around $3 \%$ protein of which $20 \%$ is whey proteins and $80 \%$ is caseins $^{64}$. Caseins which account for the majority of milk protein are represented by four distinct proteins: $\alpha_{s 1}, \alpha_{s 2}, \beta, k^{64}$. Whey proteins are a complex set of proteins that in bovine milk are mainly comprised of beta-lactoglobulin ( $(-L G)$ and alpha-lactalbumin ( $\alpha-L A)$ and small traces of blood-borne proteins such as immunoglobulins and bovine serum albumin. These two categories of proteins are broadly defined by their physical properties. Unlike caseins, whey proteins do not 
contain phosphorous but contain a large fraction of sulfur-containing amino acid residues (such as cysteine and methionine) ${ }^{64}$. These amino acids form disulfide bonds within the protein thus causing the linear amino acid structure to form a compact and well-defined globular structure that accounts for their solubility. Due to the presence of proline residues and the lack of disulfide bridges, caseins exhibit a loose, flexible structure. Caseins are also hydrophobic and are found as large colloidal particles of $50-600 \mathrm{~nm}$ in diameter, known as casein micelles ${ }^{64}$. These are supramolecular structures that hold caseins together by hydrophobic interactions and calcium phosphate ${ }^{83}$. Caseins are isolated from milk by acid or by rennet precipitation. The acid, or isoelectric, precipitation is performed at $\mathrm{pH} 4.6$, where caseins precipitate (at their isoelectric point of $\mathrm{pH} 4.6$ ) and whey proteins remain soluble ${ }^{84}$. These structural and chemical differences between the two major classes of protein in bovine milk affects the behavior of these proteins in the processing of food as well as their behaviour in the gastrointestinal tract, specifically their degree of hydrolysis by the digestive enzymes.

Over the last few decades, there has been an increase in the consumption of processed dairy products. The well-being of the dairy industry is dependent on the processing of milk into other milk based products. The processability and quality of many dairy products such as cheese mainly depends on the properties of milk protein such as caseins ${ }^{64}$. The structure of casein is crucial in the processing of milk into gelatinous substances such as yogurt and cheese ${ }^{64}$. Moreover, the heat stability of caseins allows for many milk products to be subjected to high heat treatments ${ }^{84}$. Due to their industrial importance and technological properties, there has been a growing interest in studying caseins from bovine milk ${ }^{84}$. Although much research has been undertaken in studying the casein micelles, there structure is still not well understood. 
In evaluating the structure of caseins, it is important to study the physical and chemical changes that occur during digestion in the human gastrointestinal tract ${ }^{85}$. The gold standard for investigating the human digestive process is the use of in vivo approaches. This normally involves a feeding study and acquiring serial samples from the stomach and the upper small intestine. However, these approaches are impractical for large-scale studies as they are expensive to preform, time consuming and ethically and technically difficult ${ }^{41}$. To overcome the expenses and physiological difficulties, a simple in vitro method was used in this study to simulate the gastric and intestinal conditions that occur in vivo.

This study uses a confocal microscope to visually examine the in vitro digestion of caseins by developing a simple model which allows for real time digestion of caseins in situ. This will provide a better understanding about the physiological changes that caseins undergo during digestion in the stomach and small intestine and thus help yield information about the structure of caseins during human digestion as well as provide information for designing more nutritious milk based products.

\section{Materials and methods}

\section{Samples and reagents}

Milli-Q water $(18.2 \mathrm{MO} \mathrm{cm})$ was used after purification with a Milli-Q apparatus (Millipore Corporation, Bedford, MA). Pasteurized and homogenized 0.1\% skim Beatrice milk was purchased from a local grocery store (Toronto, Ontario). Milk was kept at $4^{\circ} \mathrm{C}$ prior to in vitro digestion. Pepsin from porcine gastric mucosa (EC 3.4.23.1; catalog no. P7000; 2,500 U/mg of protein) and porcine pancreatin (catalog no. P7545; 8 x USP) were purchased from Sigma-Aldrich 
Corporation. Unless specifically stated, all chemicals were of analytical grade and were purchased from Sigma-Aldrich Corporation.

\section{Confocal Laser Scanning Microscopy}

A Zeiss LSM510 Confocal Microscope was used to visualize the microstructure of caseins during in vitro digestion. To prepare the stain for confocal microscopy, pasteurized and homogenized $0.1 \%$ skim Beatrice milk (200 $\mu \mathrm{l})$ was mixed with Nile Red [9-diethylamino-5benzo[ $\alpha$ ]phenoxazinone, $1 \mathrm{mg} / \mathrm{ml}$ in dimethyl sulfoxide, 1:100 (vol/vol)] was used to stain the hydrophobic regions of caseins. Rhodamine B [[9-(2-carboxyphenyl)-6-diethylamino-3xanthenylidene]-diethylammonium chloride, $1 \mathrm{mg} / \mathrm{ml}$ in chloroform, 1:60 (vol/vol)] was used to stain the hydrophilic regions of protein. The milk - dye mixture was mixed using a vortex at 3,000 RPM for a minute. The milk - dye mixture was filtered twice with $25 \mathrm{~mm}$ syringe filters. A10x objective lens and 20x objective lens were used during the time lapse imaging of in vitro digestion.

\subsubsection{Development of an in situ digestion chamber}

An in situ digestion chamber was designed by considering the fluid dynamics of skim milk. A milling machine, a drill machine, and an automatic saw were used in the construction of the digestion chamber. A $1 \mathrm{~mm}$ thick acrylic sheet was cut with the dimensions of $75 \mathrm{~mm} \times 25$ $\mathrm{mm}$ (for optimal positioning on the confocal microscope stage). A reaction chamber with a diameter of $1.47 \mathrm{~cm}$ was drilled (Figure A-1.). A diagonal slit was drilled from the middle of the reaction chamber to the edge of the cover slip for the insertion of simulated secretions. A small slit with a length of $1.5 \mathrm{~cm}$ was filed to alleviate the displacement of air by the milk-dye sample. 


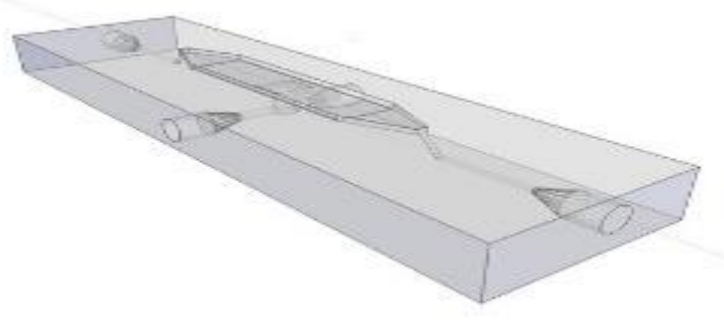

Figure A-1. Schematic of an in situ micro cell for in vitro digestion.

\section{In vitro gastric digestion}

A sample of $200 \mu$ l of the milk-dye mixture was added to the in-situ digestion chamber. The digestion chamber was placed on the microscope stage for the time lapse imaging process. The milk-dye mixture was acidified to a pH range of $1.5-2.0$ with a use of a syringe to inject 10 $\mu \mathrm{l}$ of $1 \mathrm{M} \mathrm{HCl}$ into the digestion chamber. A parallel experiment that took place away from the microscope slide was used to determine the time the sample reached a $\mathrm{pH}$ of range of $1.5-2.0$. A syringe was used to inject simulated gastric fluid containing $4 \mu \mathrm{l}$ of $3.2 \mathrm{mg} / \mathrm{ml}$ pepsin with 0.05 $\mathrm{M} \mathrm{HCl}$ into the digestion chamber. A heat stage was used to maintain the temperature of the sample at $37^{\circ} \mathrm{C}$. The time lapse image was performed for 10 minutes.

\section{In vitro intestinal digestion}

$\mathrm{HCl}$ from gastric digestion was neutralized with the addition of $6 \mathrm{M} \mathrm{NaOH}$ and the $\mathrm{pH}$ was maintained at a range of $\mathrm{pH} 7.0-7.5$ ((US Pharmacopeia, 1995). Simulated intestinal fluid containing $10 \%(\mathrm{w} / \mathrm{v})$ porcine pancreatin (with trypsin, chymotrypsin) was injected into the digestion chamber of the in-situ model for intestinal digestion. A heat stage was used to maintain the temperature of the sample at $37^{\circ} \mathrm{C}$. The time lapse image was performed for 30 minutes. 


\section{Results and discussion}

\section{Gastric digestion - casein hydrolysis}

In humans, in vitro digestion of milk proteins, begins in the stomach. During the process of digestion, casein micelles first come upon gastric acid which has a very low $\mathrm{pH}$ range of $1.0-$ 2.0. Although casein is relatively stable at high temperatures, it is sensitive to $\mathrm{pH}$ and will precipitate at its isoelectric point, $\mathrm{pH} 4.6^{64}$. The precipitation of casein in the presence of $\mathrm{HCl}$ can be observed in Figure A-2. Over the period of a minute, the $\mathrm{pH}$ dropped from $\mathrm{pH}$ of 6.77 (in native skim milk) to $\mathrm{pH} 1.5$ (from the introduction of $\mathrm{HCl}$ ) which caused the casein micelles to undergo denaturation and precipitate thus resulting in a change in shape. This is seen by Figures A-2B-D. where the shape of the coagulated caseins (casein domain) gets narrower as it is exposed for a longer duration to the low acidic environment of the stomach.

During gastric digestion in the human stomach, this clotting or precipitation of casein under the acidic environment of the stomach, results in a reduction in gastric emptying rate, which delays the release of amino acids to the small intestine ${ }^{86}$. On the other hand, milk soluble proteins such as whey are rapidly expelled out of the stomach ${ }^{86}$. 

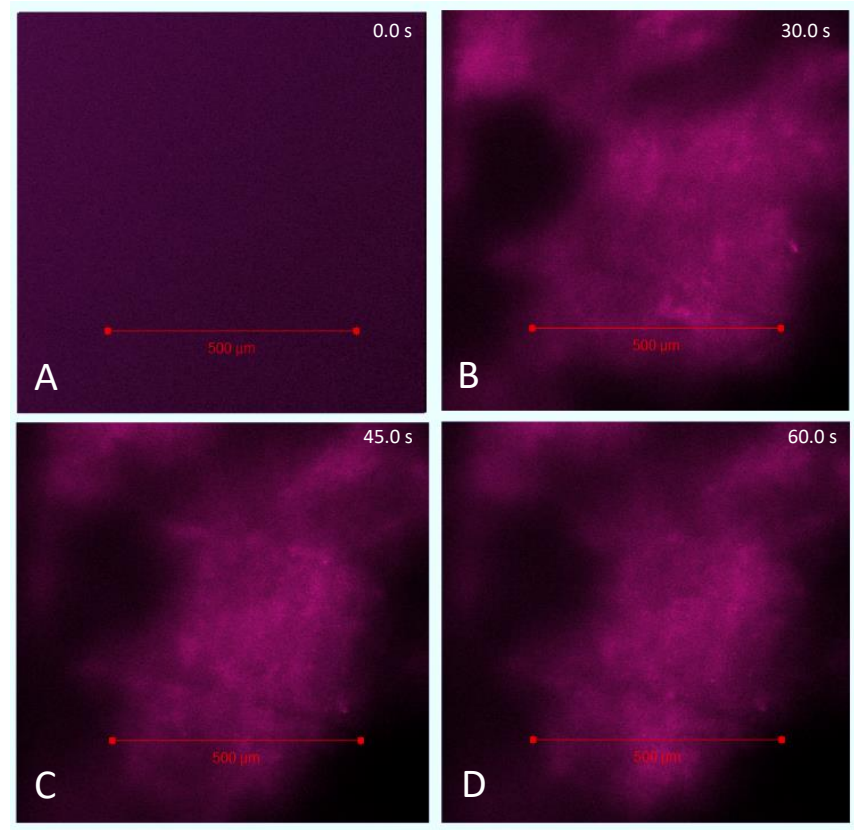

Figure A-2. Confocal laser scanning microscopy images of $0.1 \%$ skim milk (A, B, C and D) over the course of a minute after the addition of $1 \mathrm{M} \mathrm{HCl}$. Figure $A$ A. shows plain $0.1 \%$ skim milk (at time $=0.0 \mathrm{~s}$ ) before the addition of $\mathrm{HCl}$. Figures A-2B-D. show the coagulation of caseins after the addition of $1 \mathrm{M} \mathrm{HCl}$. Nile Red (A-D) was used to stain the hydrophobic regions of caseins.

Before the precipitated caseins are sent into the small intestine via gastric emptying, a digestive enzyme known as pepsin starts to hydrolyze proteins from the surface of the precipitated caseins ${ }^{87}$. Pepsin is an aspartic protease and has a preference for cleaving peptides with amino acids: phenylalanine, tryptophan, tyrosine and leucine ${ }^{88}$. Figures A-2A-D. show the structural changes of the precipitated casein domains when subjected to pepsin. Over the course of 10 minutes (Figures $A-2 A-D$ ), the size of the precipitated casein domain was reduced by half as a result of the activity of pepsin. The hydrolyzed peptides of caseins are released in to the small intestine for further digestion. A study by Gallier and Singh (2012), found that both $\beta$-casein and $\mathrm{k}$ - casein were hydrolyzed within 10 minutes of gastric digestion. However, the presence of 
both $\alpha_{s 1}, \alpha_{s 2}$ caseins were detected even after 45 minutes of gastric digestion thus suggesting a resistance to further pepsinolysis ${ }^{89}$.

Intestinal digestion - casein hydrolysis

The second stage of the in vitro digestion of caseins occurs in the small intestine. With the secretion of pancreatin (which contains proteolytic enzymes like trypsin and chymotrypsin) further digestion of caseins takes place. Trypsin cleaves the backbone of positively charged amino acids (lysine or arginine) and chymotrypsin cleaves peptides with bulky hydrophobic amino acid residues ${ }^{64}$. These proteolytic enzymes help to digest any undigested caseins and peptides. 

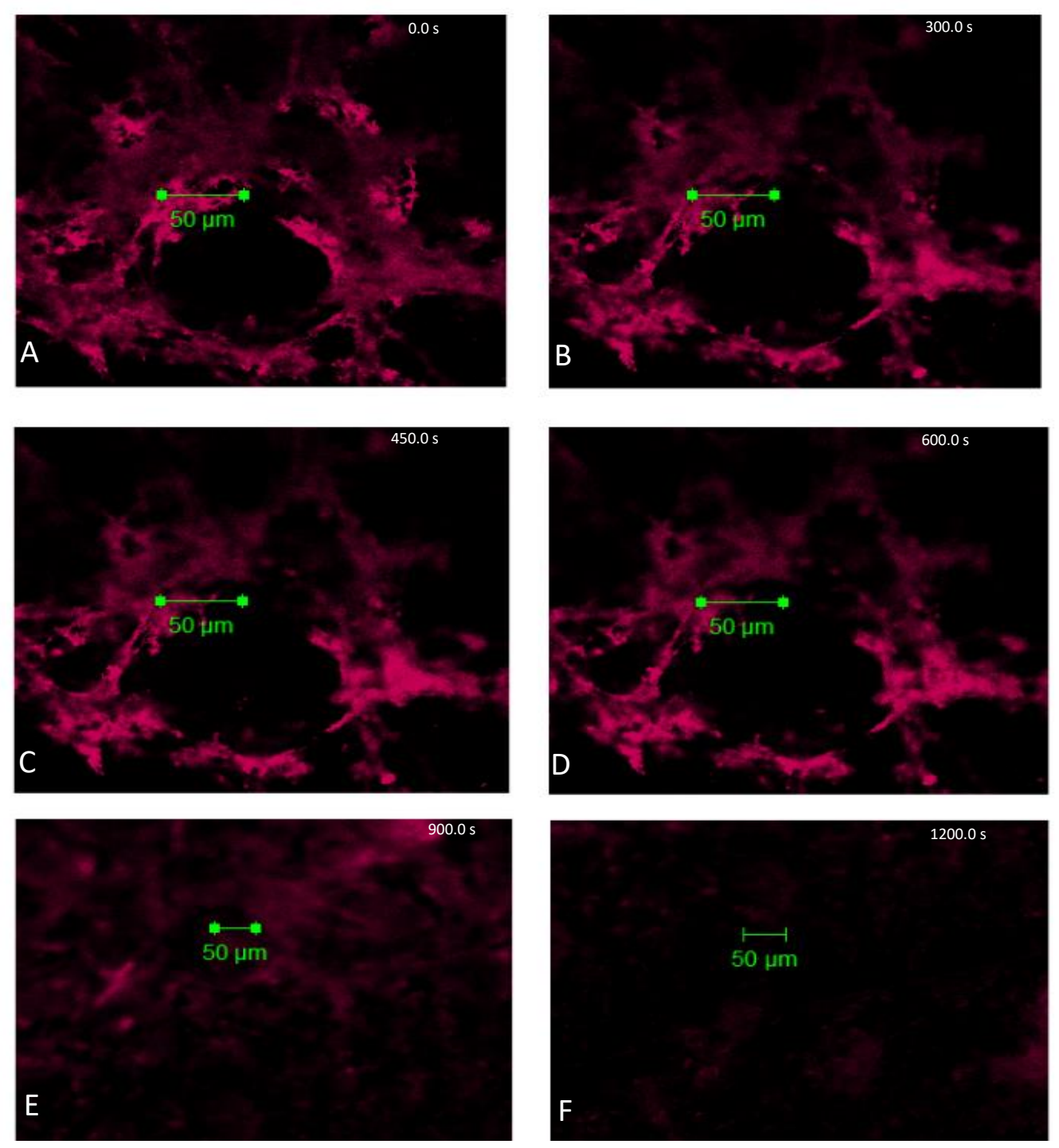

Figure A-3. Confocal laser scanning microscopy images of $0.1 \%$ skim milk ( $A, B, C$ and $D)$ subjected to simulated gastric secretion during in vitro gastric digestion. Figures $A-3 A-B$. visually show the change in structure of the casein domains during enzymatic hydrolysis by pepsin over a period of 10 minutes. These figures were captured with a use of a $20 x$ objective lens. Figures $A-3 E-F$. show images of $0.1 \%$ skim milk subjected to simulated intestinal secretion during in vitro intestinal digestion. These figures show the change in structure of the casein domains during enzymatic hydrolysis by trypsin and chymotrypsin over a period of 20 minutes. These figures were captured with a use of a 10x objective lens for a wider field of view. Nile Red (A-F) was used to stain the hydrophobic regions of caseins. The $\mathrm{pH}$ was maintained at $\mathrm{pH}$ of 7.0 during in vitro intestinal digestion. 
This is seen in Figures A-3E-F., where over the period of 20 minutes, the undigested caseins and peptides from in vitro gastric digestion are subjected to proteolysis by trypsin and chymotrypsin. At the end of the 20 minutes (Figure A-3F), most of the caseins are completely hydrolyzed. This is seen by the small, faintly fluorescing domains of caseins in Figure A-3F.

\section{Conclusion}

This study shows that the development of an in situ model for visualizing the in vitro digestion of casein can help understand the chemical and physical dynamics of caseins as they are occurring in real time. The results from using this in situ model provided similar results to what is seen in vivo since at the end of the intestinal digestion, most of the casein and residual peptides were hydrolyzed by trypsin and chymotrypsin. As such, there is potential for this in situ model for not only studying milk protein but for studying the dynamics of emulsions over time or for studying the digestion of fats. 


\section{Appendix B}

\section{In vitro GI determination of beverages}

For Coca-Cola and Fruité Fruit Punch, it was expected that since there was not too much starch to began with, most of the carbohydrate is in the form of glucose. As such, it was expected to observe a high glucose concentration in the oral phase. This was found to be the case. In the stomach, it was expected for salivary alpha amylase to be deactivated due to the very low $\mathrm{pH}$. As such, the concentration of glucose was expected to be constant. From Figure B-1., it was seen that there was a slight increase in the glucose concentrations for these beverages over the twohour residence time. This may be due to some of the sugars like maltose that were present in these drinks as they were being digested by the enzyme maltase.

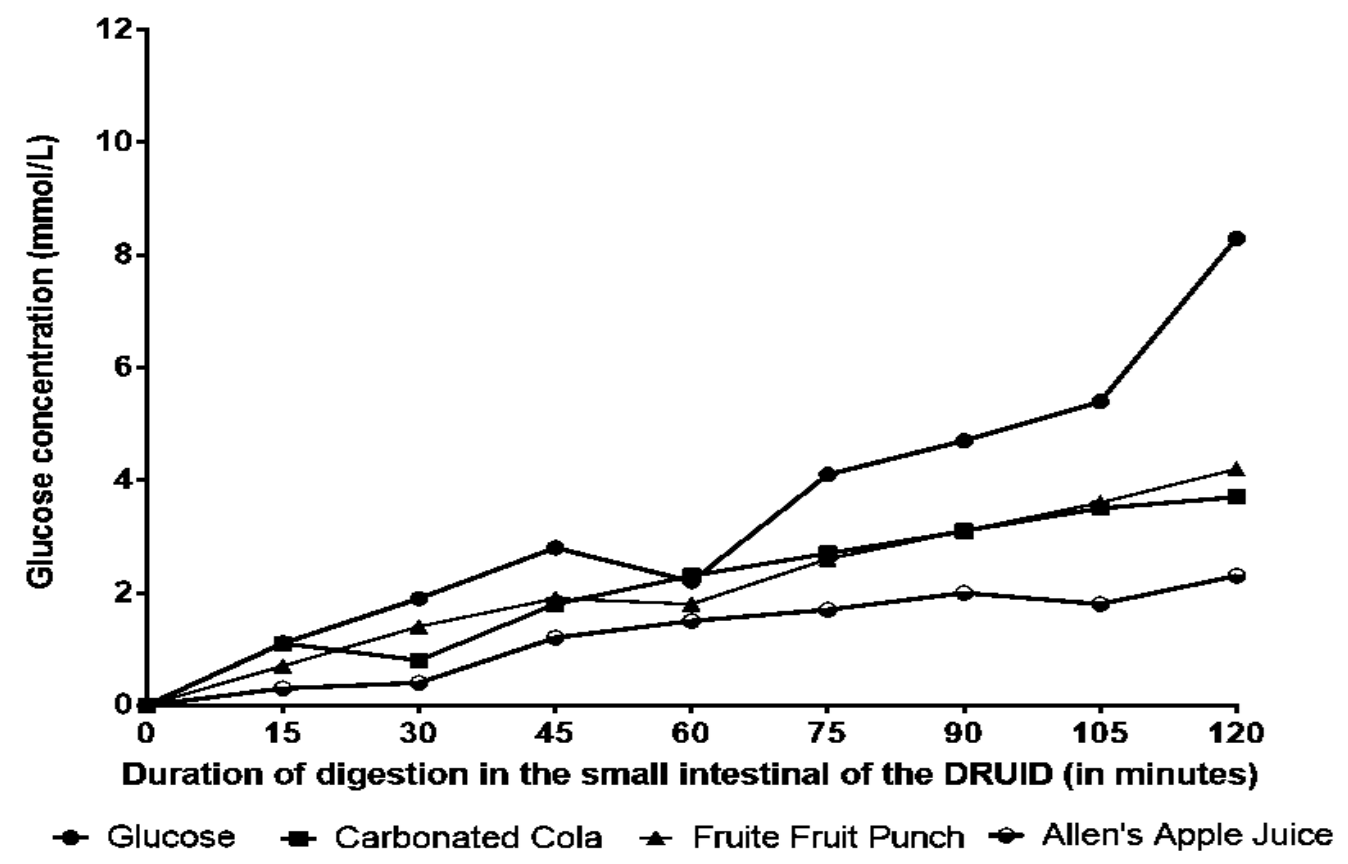

Figure B-A. Breakdown of beverages against glucose (50 $\mathrm{g}$ of available carbohydrates) in the small intestinal compartment of the DRUID. 


\section{Validation of the YSI 2700}

To validate the YSI 2700 biochemical analyzer that was extensively used to determine the glucose concentrations of samples after digestion in the DRUID, a commonly used colorimetric assay from Megazyme (GOPOD) kit was used to determine the glucose concentration of the samples after digestion in the DRUID as a way of validating the YSI 2700. Unlike the YSI 2700 biochemical analyzer that only requires to aspirate $25 \mu \mathrm{m}$ of sample to determine the glucose concentration using enzymatic digestion, the Megazyme D-Glucose (glucose oxidase/peroxidase; GOPOD) Assay Kit requires several dilutions and sample preparations in the dark. As such, this assay can lead to several errors. However, due to its inexpensive price and availability, the Megazyme D-Glucose (glucose oxidase/peroxidase; GOPOD) Assay Kit has been used in past literature ${ }^{90}$.

The validation of the YSI 2700 biochemical analyzer to the Megazyme (GOPOD) kit was compared using a Bland-Altman plot. Figure 9.2 showed that when the glucose concentrations determined from the YSI 2700 biochemical analyzer were compared to the Megazyme (GOPOD) kit for white rice, there was an agreement between the two methods (since most of the values were near the line of no difference). Moreover, Figure 9.3. showed that an agreement was also seen between the two glucose determination methods when the glucose values of an apple were compared after digestion in the DRUID.

Therefore, due to the speed of determining the glucose concentration using the YSI 2700 biochemical analyzer and the lack of error from serial dilution as well as the agreement to the 
Megazyme (GOPOD) kit for determining glucose concentrations, the YSI 2700 biochemical analyzer was preferred for glucose concentration determination.

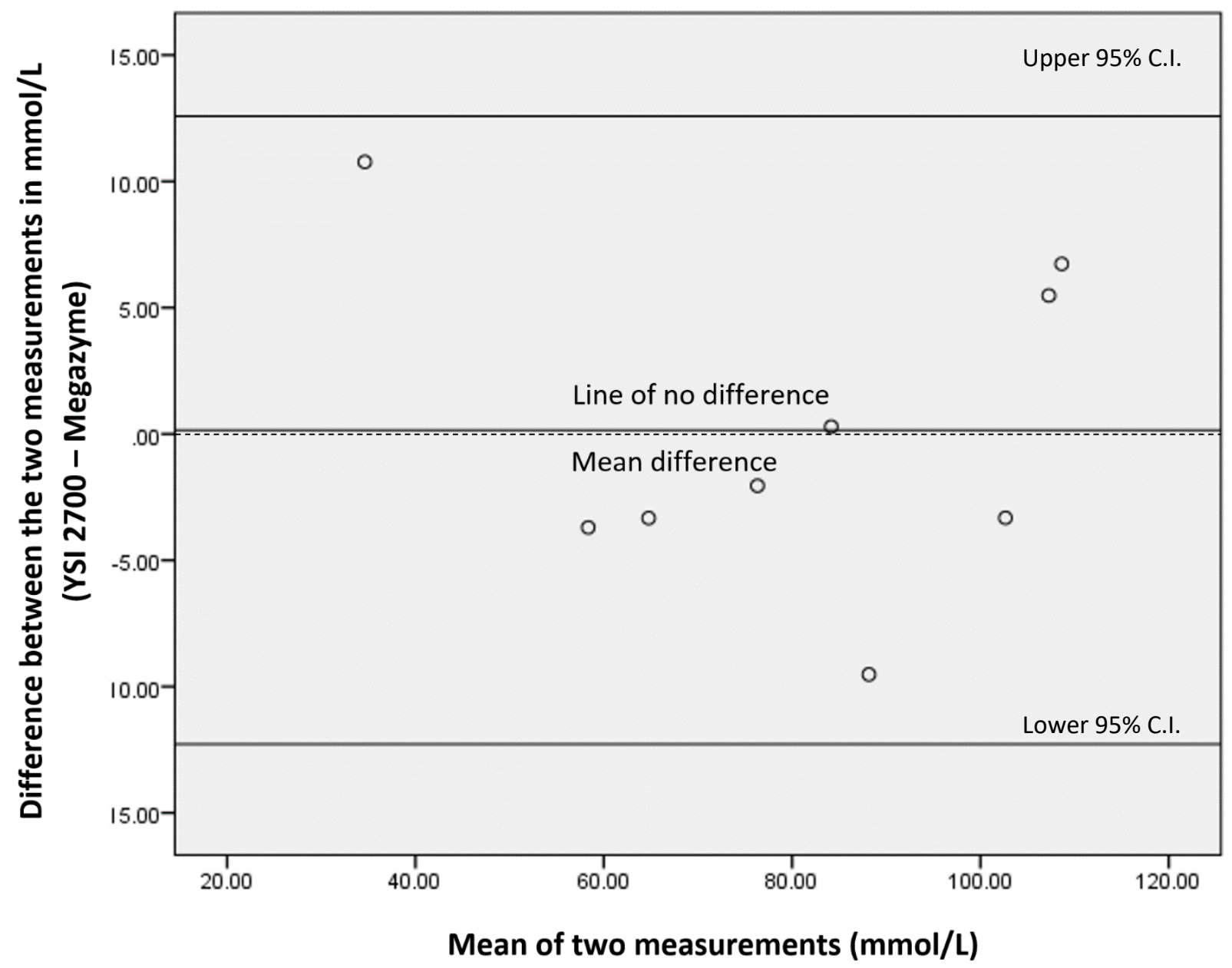

Figure B-2. Bland-Altman plot showing the agreement between the YSI 2700 biochemical analyzer and Megazyme kit for white rice. 


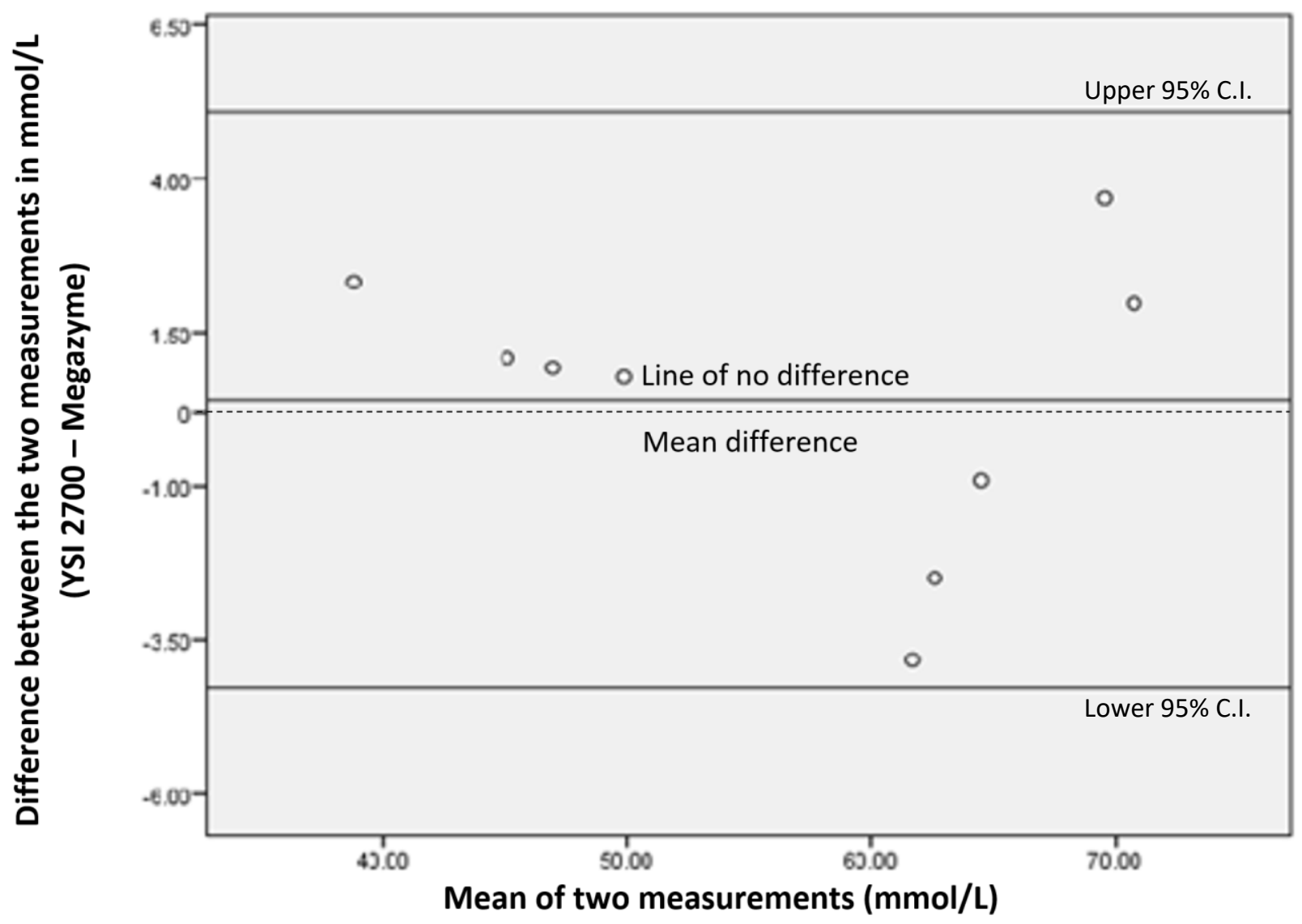

Figure B-3. Bland-Altman plot showing the agreement between the YSI 2700 biochemical analyzer and Megazyme kit for apple. 


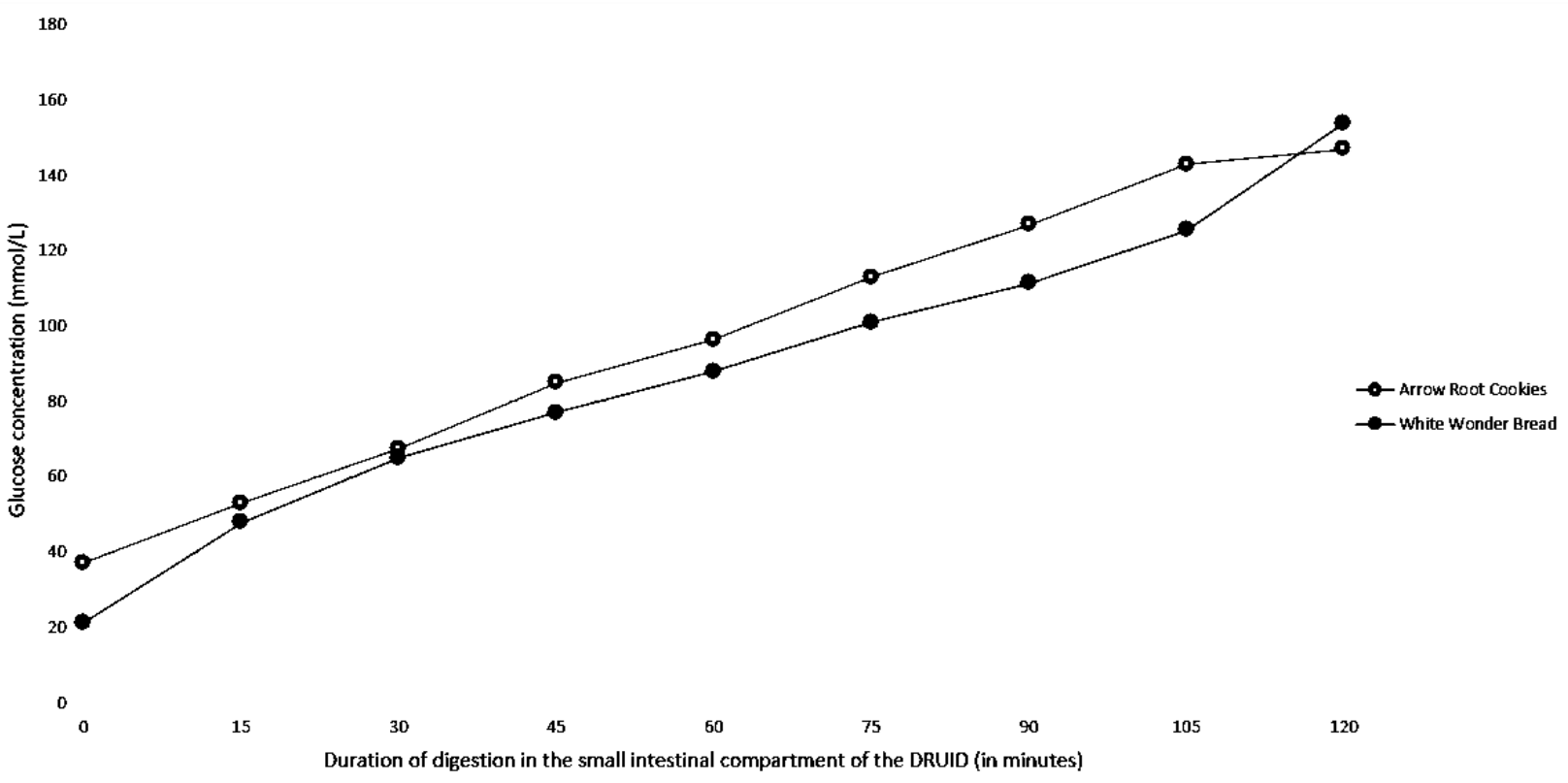

Figure B-4. Breakdown of Arrowroot cookies against white bread (50 g of available carbohydrates) in the small intestinal compartment of the DRUID.

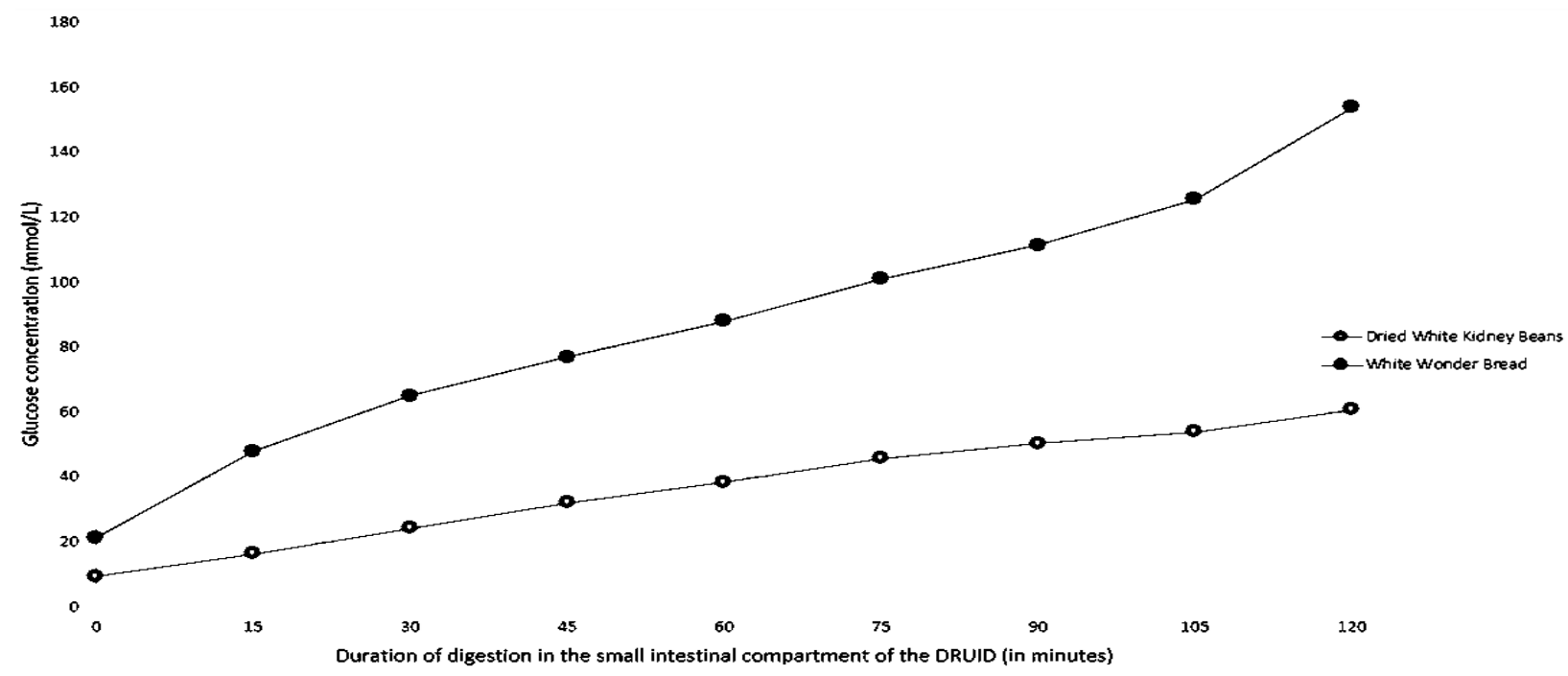

Figure B-5. Breakdown of dried white kidney beans against white bread (50 $\mathrm{g}$ of available carbohydrates) in the small intestinal compartment of the DRUID. 


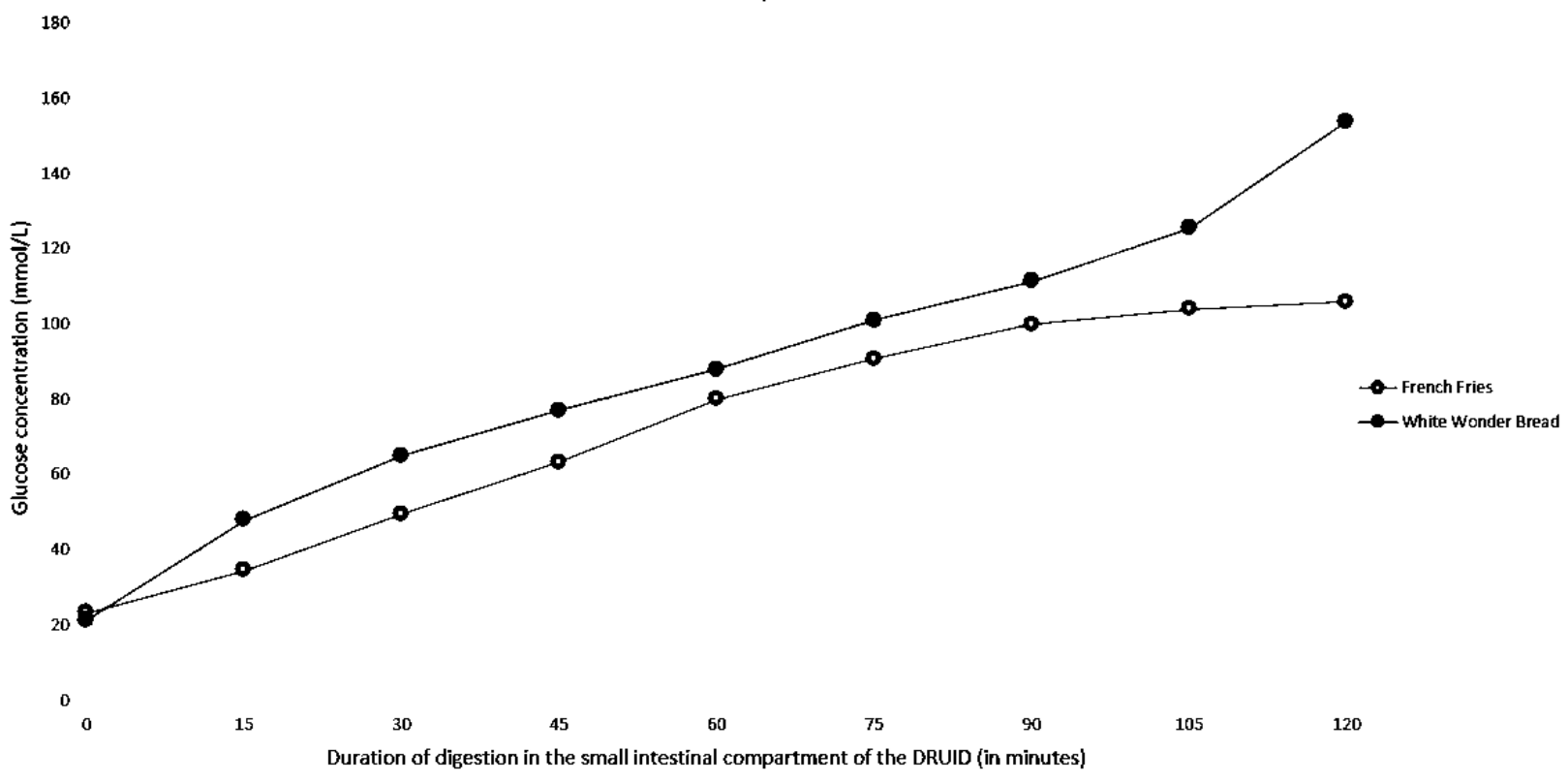

Figure B-6. Breakdown of French fries against white bread (50 g of available carbohydrates) in the small intestinal compartment of the DRUID.

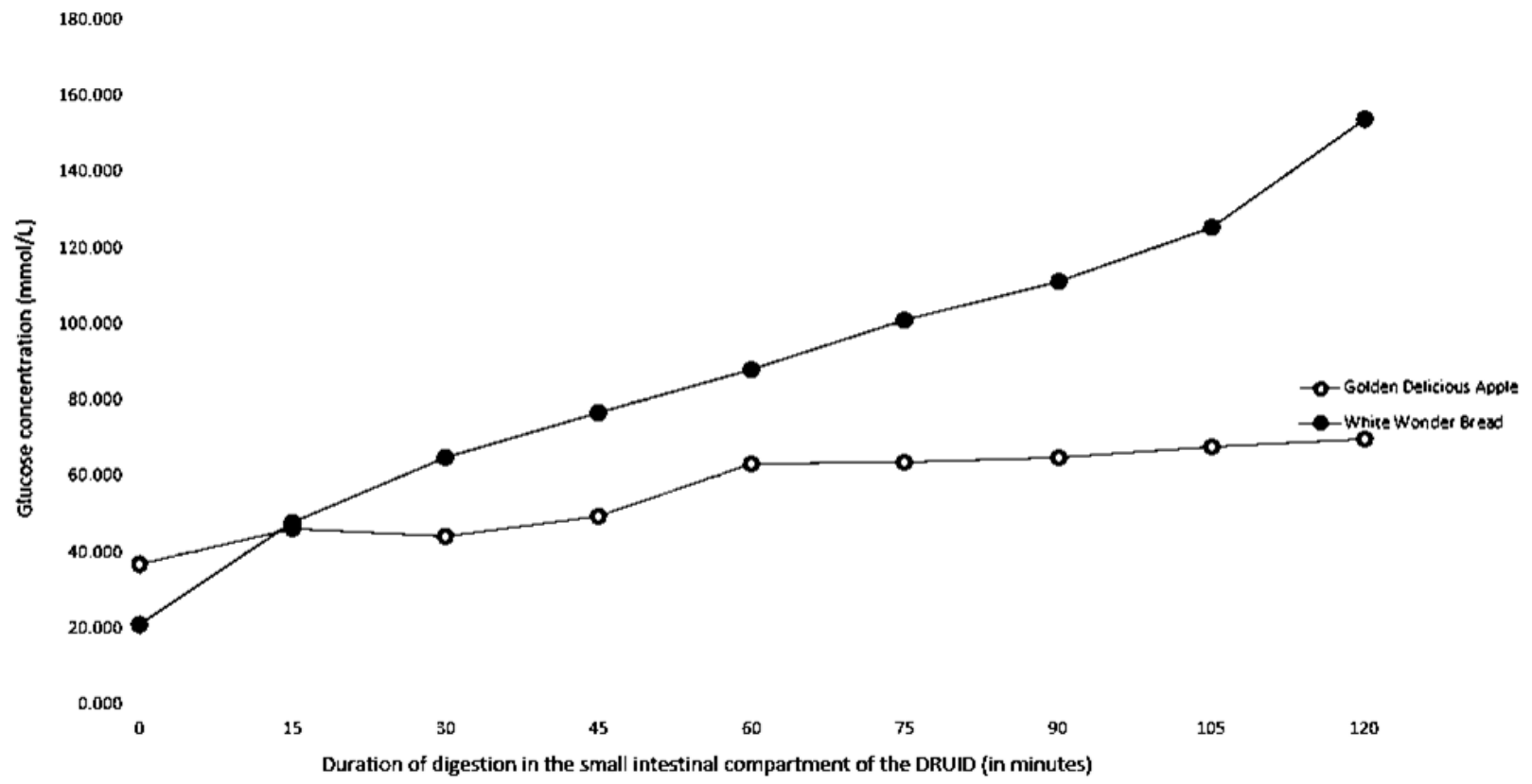

Figure B-7. Breakdown of apple against white bread (50 g of available carbohydrates) in the small intestinal compartment of the DRUID. 


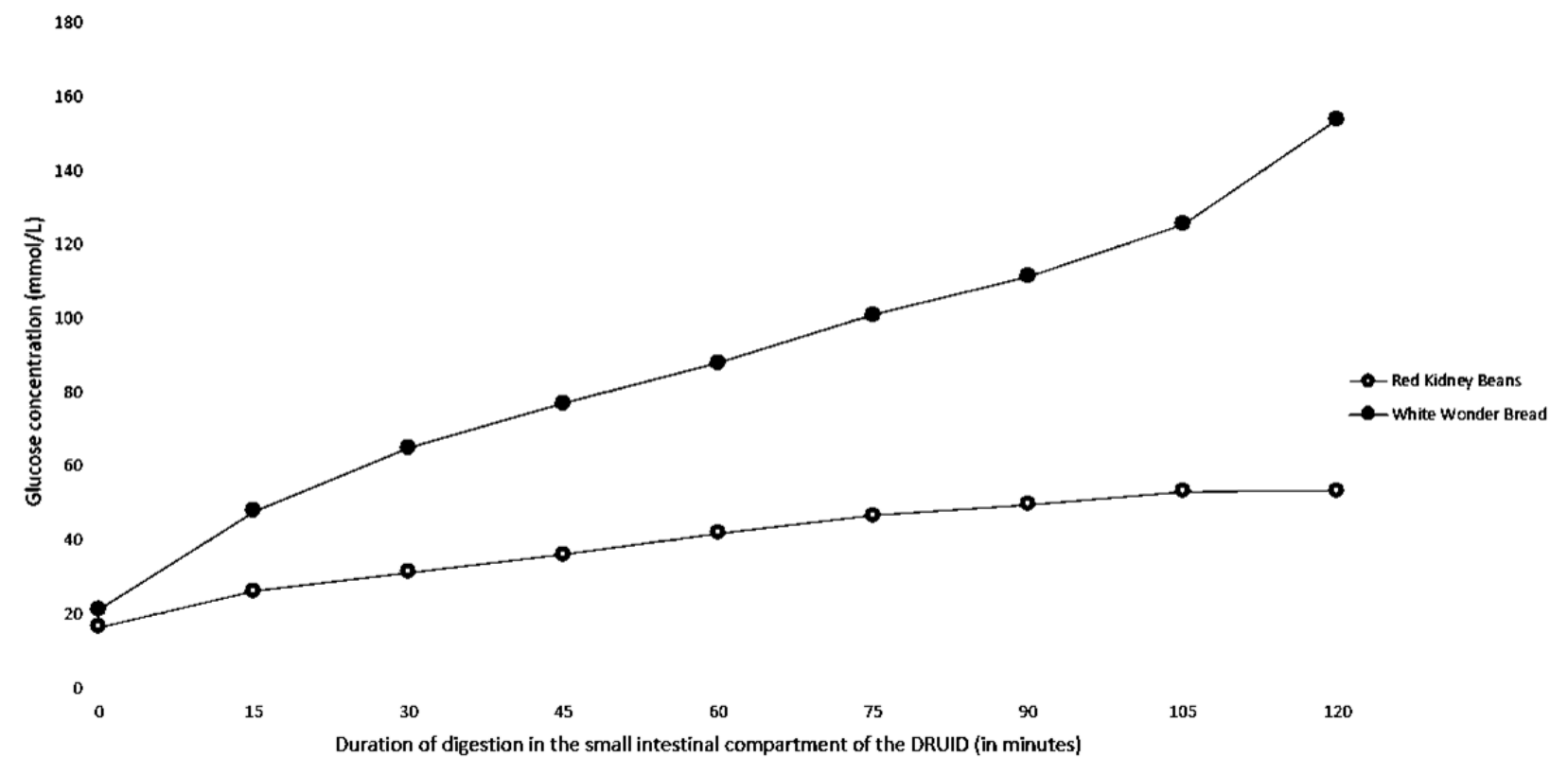

Figure B-8. Breakdown of red kidney beans against white bread (50 g of available carbohydrates) in the small intestinal compartment of the DRUID.

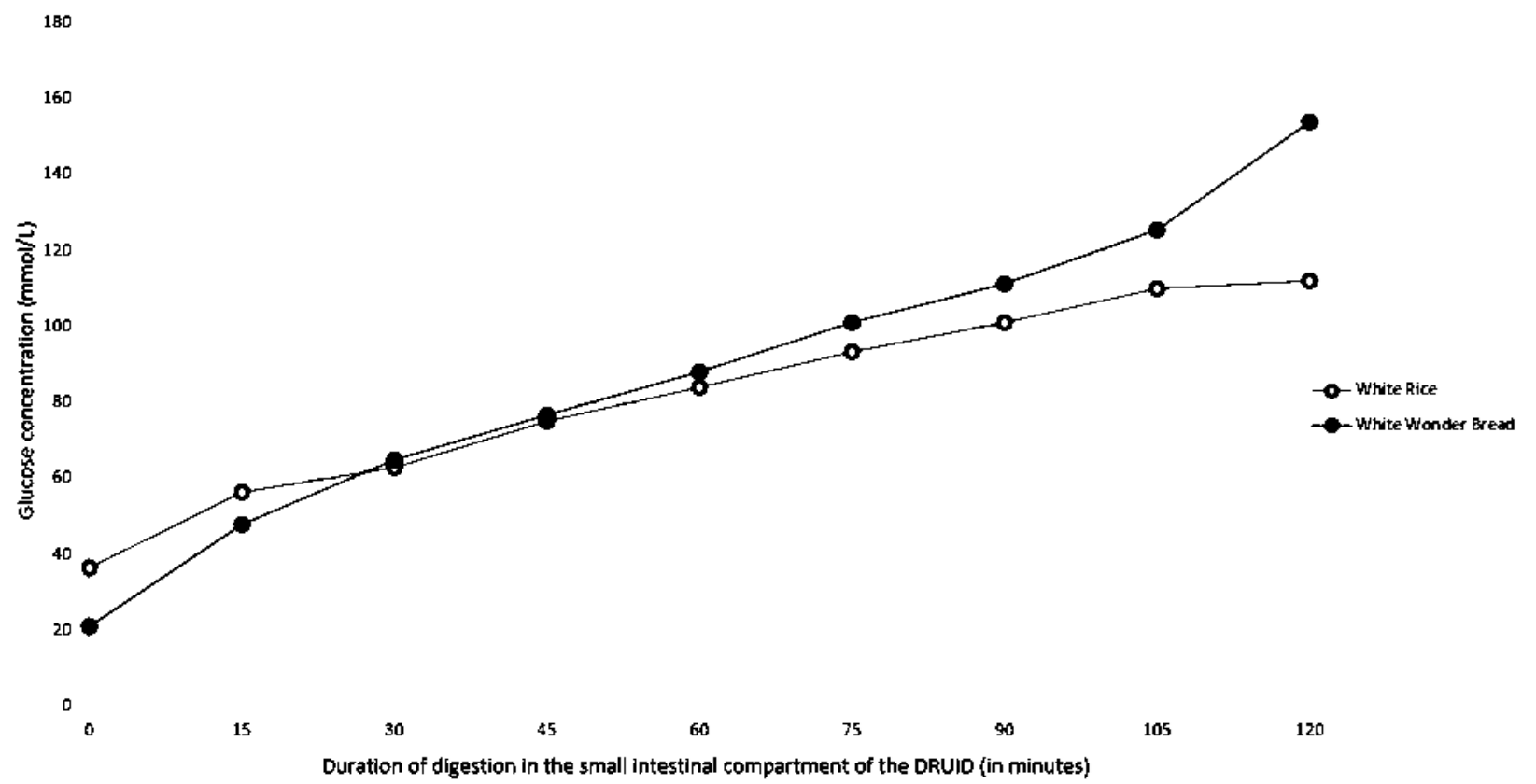

Figure B-9. Breakdown of white rice against white bread (50 g of available carbohydrates) in the small intestinal compartment of the DRUID. 


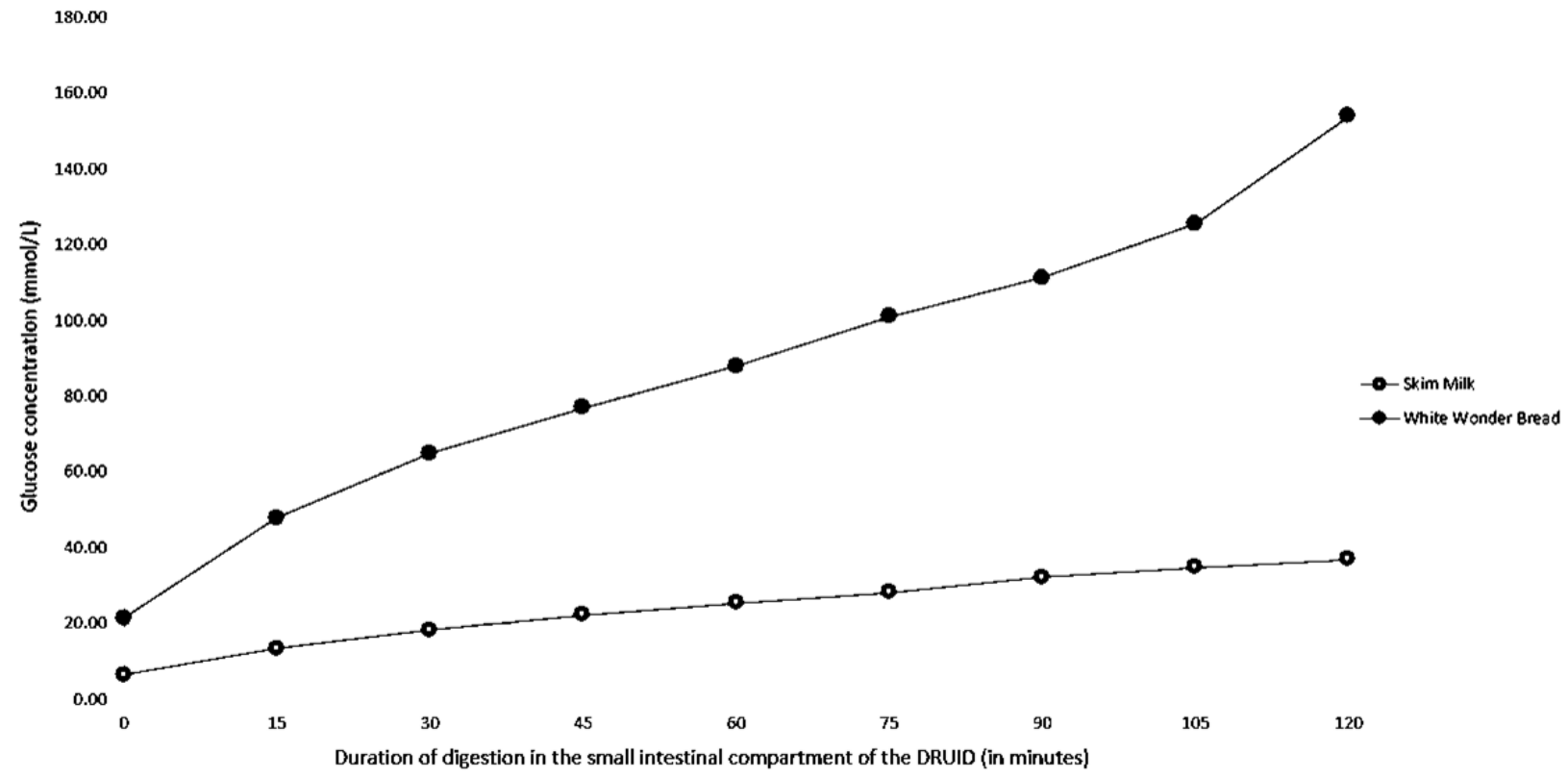

Figure B-10. Breakdown of skim milk against white bread (50 g of available carbohydrates) in the small intestinal compartment of the DRUID.

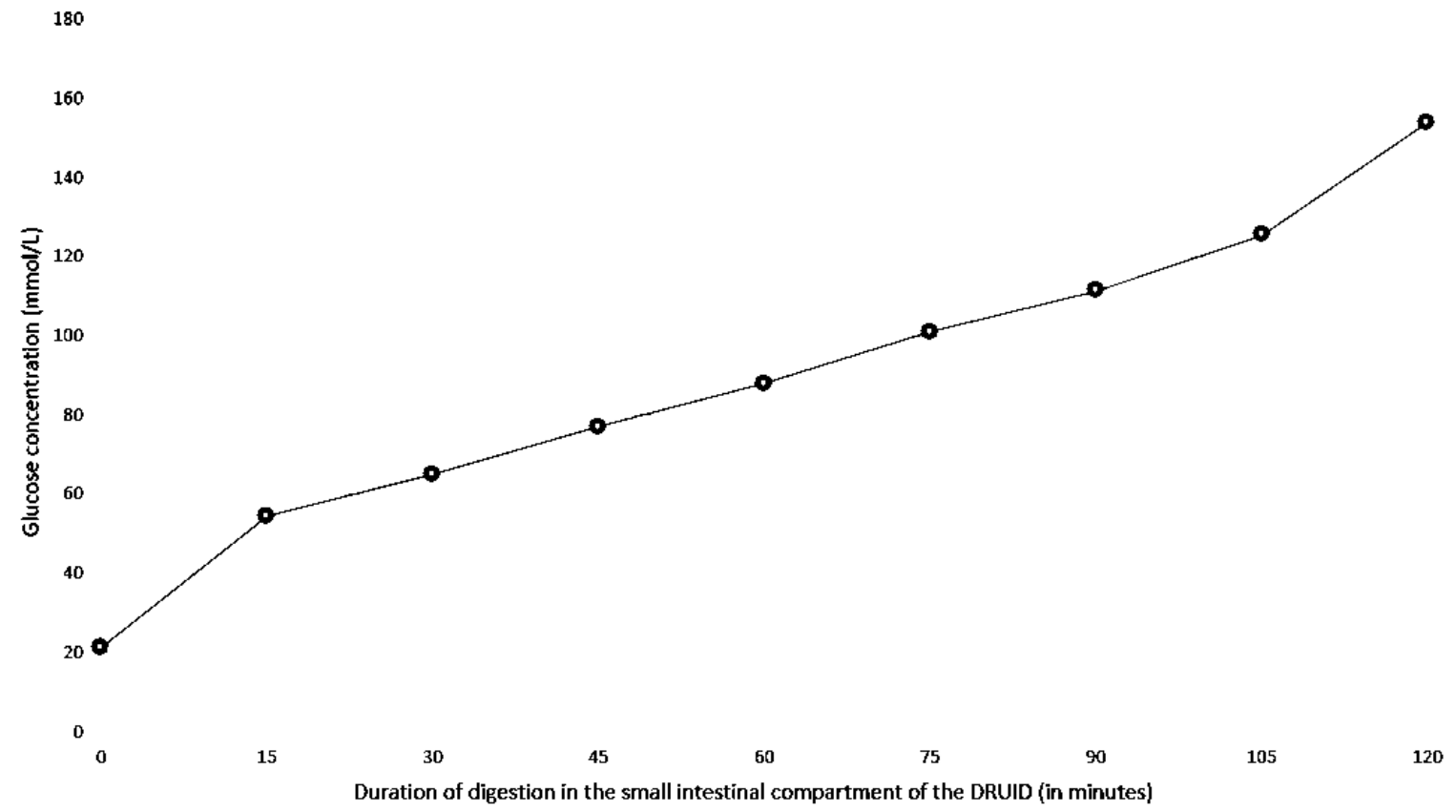

Figure B-11. Breakdown of white bread (50 g of available carbohydrates) in the small intestinal compartment of the DRUID. 


\section{References}

1. Mann J, Cummings JH, Englyst HN, et al. FAO/WHO scientific update on carbohydrates in human nutrition: conclusions. Eur J Clin Nutr. 2007;61 Suppl 1:S132-S137. doi:10.1038/sj.ejcn.1602943.

2. Keim NL. Carbohydrates. In M. E. Shils, \& Others, Mordern Nutrition Health and Disease. 10th ed. Philadelphia: Lippincott Williams \& Wilkins; 2006.

3. Venter CS SM\& VH. Labelling of foods for glyceamic index - advantages and problems. South African J Clin Nutr. 2003;16(4):118-126.

4. LN CJ\& N. The glyceamic response to isoglucogenic quantities of protein and carbohydrate. J Clin Invest. 1939;15:665-671.

5. Jenkins DJA, Wolever TMS, Taylor RH. Glycemic index of foods: A physiological basis for carbohydrate exchange. Am J Clin Nutr. 1981;34(3):362-366.

6. Augustin LS, Franceschi S, Jenkins DJ a, Kendall CWC, La Vecchia C. Glycemic index in chronic disease: a review. Eur J Clin Nutr. 2002;56(11):1049-1071.

doi:10.1038/sj.ejcn.1601454.

7. Laville M. Could glycaemic index be the basis of simple nutritional recommendations? $\mathrm{Br}$ J Nutr. 2004;91(6):803. doi:10.1079/BJN20041164.

8. Wolever TMS, Vorster HH, Björck I, et al. Determination of the glycaemic index of foods: interlaboratory study. Eur J Clin Nutr. 2003;57(3):475-482. doi:10.1038/sj.ejcn.1601551.

9. Ludwig DS. Clinical update: the low-glycaemic-index diet. Lancet. 2007;369(9565):890892. doi:10.1016/S0140-6736(07)60427-9.

10. van Bakel MME, Slimani N, Feskens EJM, et al. Methodological challenges in the application of the glycemic index in epidemiological studies using data from the European Prospective Investigation into Cancer and Nutrition. J Nutr. 2009;139(3):568575. doi:10.3945/jn.108.097121.

11. FAO/WHO. Carbohydrates in Human Nutrition. Report of a Joint FAO/WHO Expert Consultation (FAO Food and Nutrition Paper 66). Rome; 1998.

12. M M. Foods: Experimental Perspecitves. 6th Editio. London: Prentice Hall Publishers; 2009.

13. Cummings H, Englyst N. Gastrointestinal. Am J Clin Nutr. 1995;61:938S-945S.

14. Gallant DJ, Bouchet B, Buléon a, Pérez S. Physical characteristics of starch granules and susceptibility to enzymatic degradation. Eur J Clin Nutr. 1992;46 Suppl 2(JANUARY):S3S16.

15. Camire ME, Kubow S, Donnelly DJ. Potatoes and Human Health. Crit Rev Food Sci Nutr. 2009;49(10):823-840. doi:10.1080/10408390903041996.

16. Behall KM. Consumption of Both Resistant Starch and -Glucan Improves Postprandial Plasma Glucose and Insulin in Women. Diabetes Care. 2006;29(5):976-981. doi:10.2337/diacare.295976.

17. Trowell H, Southgate D., Wolever T., Leeds A, Gassull M, Jenkins D. DIETARY FIBRE REDEFINED. Lancet. 1976;307(7966):967. doi:10.1016/S0140-6736(76)92750-1.

18. Cummings JH, Stephen a M. Carbohydrate terminology and classification. Eur J Clin Nutr. 2007;61 Suppl 1:S5-S18. doi:10.1038/sj.ejcn.1602936. 
19. Lawrence RD, McCance RA. New analyses of carbohydrate foods and their application to diabetic diets. BMJ. 1929;2(3579):241-241. doi:10.1136/bmj.2.3579.241.

20. Brouns F, Bjorck I, Frayn KN, et al. Glycaemic index methodology. Nutr Res Rev. 2005;18(1):145-171. doi:10.1079/NRR2005100.

21. Scharlau D, Borowicki A, Habermann N, et al. Mechanisms of primary cancer prevention by butyrate and other products formed during gut flora-mediated fermentation of dietary fibre. Mutat Res - Rev Mutat Res. 2009;682(1):39-53.

doi:10.1016/j.mrrev.2009.04.001.

22. Englyst HN, Kingman SM, Cummings JH. Classification and measurement of nutritionally important starch fractions. Eur J Clin Nutr. 1992;46 Suppl 2:S33-50. doi:10.1111/j.17503841.2010.01627.x.

23. Bell SJ, Sears B. Low-glycemic-load diets: impact on obesity and chronic diseases. Crit Rev Food Sci Nutr. 2003;43(December 2014):357-377. doi:10.1080/10408690390826554.

24. Spieth LE, Harnish JD, Lenders CM, et al. A low-glycemic index diet in the treatment of pediatric obesity. Arch Pediatr Adolesc Med. 2000;154(9):947-951.

https://www.scopus.com/inward/record.uri?eid=2-s2.0-

0033821182\& partnerID=40\&md5=3425fed680edfac85de5d6512a3f444f.

25. Blaak EE, Saris WHM. Health aspects of various digestible carbohydrates. Nutr Res. 1995;15(10):1547-1573. doi:10.1016/0271-5317(95)02027-S.

26. Ludwig DS. The glycemic index: physiological mechanisms relating to obesity, diabetes, and cardiovascular disease. JAMA. 2002;287(18):2414-2423.

doi:10.1001/jama.287.18.2414.

27. Brand-Miller JC, Holt SHA, Pawlak DB, McMillan J. Glycemic index and obesity. In: American Journal of Clinical Nutrition. Vol 76. ; 2002. doi:10.3945/an.114.007120.sugars.

28. Thibodeau GA PK. Anthony's Textbook of Anatomy \& Physiology. 18th ed. St. Louis: Mosby; 2006.

29. Evans DF, Pye G, Bramley R, Clark a G, Dyson TJ, Hardcastle JD. Measurement of gastrointestinal pH profiles in normal ambulant human subjects. Gut. 1988;29(8):10351041. doi:10.1136/gut.29.8.1035.

30. Mese H, Matsuo R. Salivary secretion, taste and hyposalivation. J Oral Rehabil. 2007;34(10):711-723. doi:10.1111/j.1365-2842.2007.01794.x.

31. Humphrey SP, Williamson RT. A review of saliva: normal composition, flow, and function. J Prosthet Dent. 2001;85(2):162-169. doi:10.1067/mpr.2001.113778.

32. Mackie DA, Pangborn RM. Mastication and its influence on human salivary flow and alpha-amylase secretion. Physiol Behav. 1990;47(3):593-595. doi:10.1016/00319384(90)90131-M.

33. Kong F, Singh RP. A Human Gastric Simulator (HGS) to Study Food Digestion in Human Stomach. J Food Sci. 2010;75(9). doi:10.1111/j.1750-3841.2010.01856.x.

34. Marciani L, Gowland PA, Spiller RC, et al. Effect of meal viscosity and nutrients on satiety, intragastric dilution, and emptying assessed by MRI. Am J Physiol Gastrointest Liver Physiol. 2001;280(6):G1227-G1233.

35. Beck IT. The role of pancreatic enzymes in digestion. Am J Clin Nutr. 1973;26(3):311-325. doi:10.1177/003803857300700127.

36. Johnson IT, Gee JM. Effect of gel-forming gums on the intestinal unstirred layer and sugar 
transport in vitro. Gut. 1981;22(5):398-403.

http://www.scopus.com/inward/record.url?eid=2-s2.0-

0019419013\&partnerID=tZOtx3y1.

37. Rosenblum JL, Irwin CL, Alpers DH. Starch and glucose oligosaccharides protect salivarytype amylase activity at acid pH. Am J Physiol. 1988;254(5 Pt 1):G775-G780.

38. Wright EM, Martín MG, Turk E. Intestinal absorption in health and disease--sugars. Best Pract Res Clin Gastroenterol. 2003;17(6):943-956. doi:10.1016/S1521-6918(03)00107-0.

39. Buonocore V, Poerio E. Interaction of Tenebrio molitor L. $\alpha$-amylase with a wheat flour protein inhibitor. FEBS Lett. 1976;67(2):202-206. doi:10.1016/0014-5793(76)80366-3.

40. Martins JC, Enassar M, Willem R, Wieruzeski J-M, Lippens G, Wodak SJ. Solution structure of the main $\alpha$-amylase inhibitor from amaranth seeds. Eur J Biochem. 2001;268(8):23792389. doi:10.1046/j.1432-1327.2001.02118.x.

41. Boisen S, Eggum BO. Critical evaluation of in vitro methods for estimating digestibility in simple-stomach animals. Nutr Res Rev. 1991;4(1):141-162. doi:10.1079/NRR19910012.

42. Parada J, Aguilera JM. Food microstructure affects the bioavailability of several nutrients. J Food Sci. 2007;72(2). doi:10.1111/j.1750-3841.2007.00274.x.

43. Minekus $M$, Alminger $M$, Alvito $P$, et al. A standardised static in vitro digestion method suitable for food - an international consensus. Food Funct. 2014;5(6):1113-1124. doi:10.1039/c3fo60702j.

44. Fernández-García E, Carvajal-Lérida I, Pérez-Gálvez A. In vitro bioaccessibility assessment as a prediction tool of nutritional efficiency. Nutr Res. 2009;29(11):751-760. doi:10.1016/j.nutres.2009.09.016.

45. Vardakou M, Mercuri A, Barker SA, Craig DQM, Faulks RM, Wickham MSJ. Achieving Antral Grinding Forces in Biorelevant In Vitro Models: Comparing the USP Dissolution Apparatus II and the Dynamic Gastric Model with Human In Vivo Data. AAPS PharmSciTech. 2011;12(2):620-626. doi:10.1208/s12249-011-9616-z.

46. Golding $M$, Wooster TJ. The influence of emulsion structure and stability on lipid digestion. Curr Opin Colloid Interface Sci. 2010;15(1-2):90-101. doi:10.1016/j.cocis.2009.11.006.

47. Ji YY, Xiao DC. GIT physicochemical modeling - A critical review. Int J Food Eng. 2006;2(4). https://www.scopus.com/inward/record.uri?eid=2-s2.033750910000\&partnerID=40\&md5=54dcb2feafc647210be3a341913493ea.

48. Minekus M, Marteau P, Havenaar R, Huis in 't Veld JHJ. A multicompartmental dynamic computer-controlled model simulating the stomach and small intestine. Altern to Lab Anim. 1995;23(August 2015):197-209.

49. Fontijn-Tekamp FA, Slagter AP, Van\&apos;t Hof MA, Geertman ME, Kalk W. Bite forces with mandibular implant-retained overdentures. In: Journal of Dental Research. Vol 77. ; 1998:1832-1839. http://www.scopus.com/inward/record.url?eid=2-s2.00032223618\&partnerID=tZOtx3y1.

50. Peyron MA, Mishellany A, Woda A. Particle size distribution of food boluses after mastication of six natural foods. J Dent Res. 2004;83(7):578-582. http://www.scopus.com/inward/record.url?eid=2-s2.04143123683\&partnerID=tZOtx3y1.

51. Versantvoort CHM, Oomen AG, Van de Kamp E, Rompelberg CJM, Sips AJAM. 
Applicability of an in vitro digestion model in assessing the bioaccessibility of mycotoxins from food. Food Chem Toxicol. 2005;43(1):31-40. doi:10.1016/j.fct.2004.08.007.

52. Pedersen A, Bardow A, Jensen SB, Nauntofte B. Saliva and gastrointestinal functions of taste, mastication, swallowing and digestion. Oral Dis. 2002;8(3):117-129.

doi:10.1034/j.1601-0825.2002.02851.x.

53. Tyssandier V, Reboul E, Dumas JF, et al. Processing of vegetable-borne carotenoids in the human stomach and duodenum. Am J Physiol - Gastrointest Liver Physiol. 2003;284(6 476). http://www.scopus.com/inward/record.url?eid=2-s2.00037910167\&partnerID=tZOtx3y1.

54. Lin HC, Prather C, Fisher RS, et al. Measurement of Gastrointestinal Transit. Dig Dis Sci. 2005;50(6):989-1004. doi:10.1007/s10620-005-2694-6.

55. Dressman JB, Berardi RR, Dermentzoglou LC, et al. Upper gastrointestinal (GI) pH in young, healthy men and women. Pharm Res. 1990;7(7):756-761.

doi:10.1023/A:1015827908309.

56. Carriere F, Moreau H, Raphel V, et al. Purification and biochemical characterization of dog gastric lipase. Eur J Biochem. 1991;202(1):75-83.

http://www.scopus.com/inward/record.url?eid=2-s2.00025948932\&partnerID=tZOtx3y1.

57. Akerberg a K, Liljeberg HG, Granfeldt YE, Drews a W, Bj-orck IM. An in vitro method, based on chewing, to predict resistant starch content in foods allows parallel determination of potentially available starch and dietary fiber. J Nutr. 1998;128(3):651660.

58. Duodu KG, Taylor JRN, Belton PS, Hamaker BR. Factors affecting sorghum protein digestibility. J Cereal Sci. 2003;38(2):117-131. doi:10.1016/S0733-5210(03)00016-X.

59. Woolnough JW, Monro JA, Brennan CS, Bird AR. Simulating human carbohydrate digestion in vitro : a review of methods and the need for standardisation. Int J Food Sci Technol. 2008;43(12):2245-2256. doi:10.1111/j.1365-2621.2008.01862.x.

60. Almaa $\mathrm{H}, \mathrm{Holm} \mathrm{H}$, Langsrud T, Flengsrud R, Vegarud GE. In vitro studies of the digestion of caprine whey proteins by human gastric and duodenal juice and the effects on selected microorganisms. Br J Nutr. 2006;96(3):562-569. doi:10.1079/BJN20061739.

61. J.T. Winzeler DM. Monitoring and Control of Nutrients and Byproducts, Biomedical Products. 1997.

62. Fernandes $G$, Velangi A, Wolever TMS. Glycemic index of potatoes commonly consumed in North America. J Am Diet Assoc. 2005;105(4):557-562. doi:10.1016/j.jada.2005.01.003.

63. Foster-Powell K, Holt SHA, Brand-Miller JC. International table of glycemic index and glycemic load values: 2002. Am J Clin Nutr. 2002;76(1):5-56. http://www.ncbi.nlm.nih.gov/pubmed/12081815.

64. Farrell HM, Jimenez-Flores R, Bleck GT, et al. Nomenclature of the Proteins of Cows' Milk-Sixth Revision. J Dairy Sci. 2004;87(6):1641-1674. doi:10.3168/jds.S00220302(04)73319-6.

65. Miles MJ, Morris VJ, Orford PD, Ring SG. The roles of amylose and amylopectin in the gelation and retrogradation of starch. 1985;135(2):271-281. doi:10.1016/S00086215(00)90778-X.

66. Ring SG, Gee JM, Whittam M, Orford P, Johnson IT. Resistant starch: Its chemical form in 
foodstuffs and effect on digestibility in vitro. Food Chem. 1988;28(2):97-109. doi:10.1016/0308-8146(88)90139-2.

67. Lin MHA, Wu MC, Lu S, Lin J. Glycemic index, glycemic load and insulinemic index of Chinese starchy foods. World J Gastroenterol. 2010;16(39):4973-4979. doi:10.3748/wjg.v16.i39.4973.

68. Bahado-Singh PS, Riley CK, Wheatley AO, Lowe HIC. Relationship between processing method and the glycemic indices of ten sweet potato (Ipomoea batatas) cultivars commonly consumed in Jamaica. J Nutr Metab. 2011;2011. doi:10.1155/2011/584832.

69. Atkinson FS, Foster-Powell K, Brand-Miller JC. International tables of glycemic index and glycemic load values: 2008. Diabetes Care. 2008;31(12):2281-2283. doi:10.2337/dc081239.

70. Mikkelsen PB, Toubro S, Astrup A. Effect of fat-reduced diets on 24-h energy expenditure: Comparisons between animal protein, vegetable protein, and carbohydrate. Am J Clin Nutr. 2000;72(5):1135-1141.

71. Behall KM, Scholfield DJ, Canary J. Effect of starch structure on glucose and insulin responses in adults. Am J Clin Nutr. 1988;47(3):428-432. https://www.scopus.com/inward/record.uri?eid=2-s2.00023899808\&partnerID=40\&md5=1f0ddf4cb43be3360993c9518ee5fbb0.

72. Aston LM, Jackson D, Monsheimer S, et al. Developing a methodology for assigning glycaemic index values to foods consumed across Europe. Obes Rev. 2010;11(1):92-100. doi:10.1111/j.1467-789X.2009.00690.x.

73. Argyri K, Athanasatou A, Bouga M, Kapsokefalou M. The Potential of an in Vitro Digestion Method for Predicting Glycemic Response of Foods and Meals. Nutrients. 2016;8(4):209. doi:10.3390/nu8040209.

74. Englyst HN, Veenstra J, Hudson GJ. Measurement of rapidly available glucose (RAG) in plant foods: a potential in vitro predictor of the glycaemic response. Br J Nutr. 1996;75(3):327-337. doi:10.1079/BJN19960137.

75. Magaletta RL, DiCataldo SN, Liu D, Li HL, Borwankar RP, Martini MC. In vitro method for predicting glycemic index of foods using simulated digestion and an artificial neural network. Cereal Chem. 2010;87(4):363-369. doi:10.1094/CCHEM-87-4-0363.

76. Karamanlis A, Chaikomin R, Doran S, et al. Effects of protein on glycemic and incretin responses and gastric emptying after oral glucose in healthy subjects. Am J Clin Nutr. 2007;86(5):1364-1368. doi:86/5/1364 [pii].

77. Ou S, Kwok K, Li Y, Fu L. In Vitro Study of Possible Role of Dietary Fiber in Lowering Postprandial Serum Glucose. J Agric Food Chem. 2001;49(2):1026-1029. doi:10.1021/jf000574n.

78. Gentilcore $\mathrm{D}$, Chaikomin $\mathrm{R}$, Jones $\mathrm{KL}$, et al. Effects of fat on gastric emptying of and the glycemic, insulin, and incretin responses to a carbohydrate meal in type 2 diabetes. $J$ Clin Endocrinol Metab. 2006;91(6):2062-2067. doi:10.1210/jc.2005-2644.

79. Collier G, O'Dea K. The effect of coingestion of fat on the glucose, insulin, and gastric inhibitory polypeptide responses to carbohydrate and protein. Am J Clin Nutr. 1983;37(6):941-944.

80. Crowe TC, Seligman SA, Copeland L. Inhibition of enzymic digestion of amylose by free fatty acids in vitro contributes to resistant starch formation. J Nutr. 2000;130(8):2006- 
2008. https://www.scopus.com/inward/record.uri?eid=2-s2.0-

0033933730\&partner ID=40\&md5=5e2696106c023d3cc56ef5773182e04a.

81. Miller GD JJ\& ML. The Importance of Milk and Milk Products in the Diet In: Handbook of Dairy Foods and Nutrition. 2nd ed. (I W, ed.). Boca Raton, Florida, USA.: CRC Press; 2000.

82. Agostoni $C$, Carratù $B$, Boniglia $C$, et al. Free amino acid content in standard infant formulas: comparison with human milk. J Am Coll Nutr. 2000;19(4):434-438. http://eutils.ncbi.nlm.nih.gov/entrez/eutils/elink.fcgi?dbfrom=pubmed\&amp;id=109634 61\&amp;retmode=ref\&amp;cmd=prlinks.

83. Rollema HS. Casein Association and Micelle Formation, In Advanced Dairy Chemistry, Vol. 1: Proteins (Ed. P.F. Fox). Essex: Elsevier Science Publisher; 1992.

84. Fox, P.F. and McSweeney PL. Dairy Chemistry and Biochemistry. London: Blackie Academic \& Professional; 1998.

85. Fuller MF, Tomé D. In vivo determination of amino acid bioavailability in humans and model animals. J AOAC Int. 2005;88(3):923-934.

86. Mahé S, Roos N, Benamouzig R, et al. Gastrojejunal kinetics and the digestion of [15N] $\beta$ lactoglobulin and casein in humans: The influence of the nature and quantity of the protein. Am J Clin Nutr. 1996;63(4):546-552.

87. Chabance B, Marteau P, Rambaud JC, et al. Casein peptide release and passage to the blood in humans during digestion of milk or yogurt. Biochimie. 1998;80(2):155-165. doi:10.1016/S0300-9084(98)80022-9.

88. Fujimoto Z, Fujii Y, Kaneko S, Kobayashi H, Mizuno H. Crystal structure of aspartic proteinase from Irpex lacteus in complex with inhibitor pepstatin. $J$ Mol Biol. 2004;341(5):1227-1235. doi:10.1016/j.jmb.2004.06.049.

89. Gallier S, Ye A, Singh H. Structural changes of bovine milk fat globules during in vitro digestion. J Dairy Sci. 2012;95(7):3579-3592. doi:10.3168/jds.2011-5223.

90. Chau C-F, Huang Y-L, Lee M-H. In Vitro Hypoglycemic Effects of Different Insoluble FiberRich Fractions Prepared from the Peel of Citrus Sinensis L. cv. Liucheng. J Agric Food Chem. 2003;51(22):6623-6626. doi:10.1021/jf034449y. 